\title{
Comparing Two Different Initial Conditions AAMQS and Quartic Action in Illustrating the Effect of Valence Color Charges in High-Energy $p A$ Collisions at Forward Rapidity
}

\author{
Ye-Yin Zhao, Ya-Hui Chen, Ya-Qin Gao, and Fu-Hu Liu \\ Institute of Theoretical Physics, Shanxi University, Taiyuan, Shanxi 030006, China \\ Correspondence should be addressed to Fu-Hu Liu; fuhuliu@163.com
}

Received 7 November 2012; Revised 29 January 2013; Accepted 14 February 2013

Academic Editor: Terry Sloan

Copyright ( 2013 Ye-Yin Zhao et al. This is an open access article distributed under the Creative Commons Attribution License, which permits unrestricted use, distribution, and reproduction in any medium, provided the original work is properly cited.

The inclusive particle productions in proton-proton $(p p)$ and deuton-gold $(d+\mathrm{Au})$ collisions at forward rapidity at the Relativistic Heavy Ion Collider (RHIC) energy are studied in the framework of the color glass condensate (CGC) theory by using two different initial conditions: AAMQS (Albacete-Armesto-Milhano-Quiroga-Salgado) and quartic action. Then, the results obtained by the two different initial conditions in illustrating the effect of valence color charges in high-energy proton-nucleus $(p A)$ collisions at forward energy are compared. Meanwhile, the inclusive particle productions in $p A$ collisions at forward rapidity at the Large Hadron Collider (LHC) energies are predicted. The main dynamical input in our calculations is the use of solutions of the running coupling Balitsky-Kovchegov equation tested in electron-proton $(e p)$ collision data. Particle production is computed via the hybrid formalisms to obtain spectra and yields. These baseline predictions are useful for testing the current understanding of the dynamics of very strong color fields against the upcoming LHC data.

\section{Introduction}

As a self-consistent effective perturbative quantum chromodynamics (QCD) theory at high energy (or small momentum fraction $x$ ), the color glass condensate (CGC) framework has been successfully applied to many QCD processes in highenergy collisions $[1,2]$. Generally, in the initial state, the collisions involve at least one hadron or nucleus. Including the needed unitarity corrections, the Jalilian-Marian-IancuMcLerran-Weigert-Leonidov-Kovner (JIMWLK) renormalization group equations [3-8] capture the dynamical features of the CGC, which provides the tools to perform such resummation, although the precise prescription for the resummation may vary from process to process or colliding system.

In the framework of the CGC, as an instrumental tool, the dipole model formulation of deep inelastic scattering (DIS) allows for a relatively simple implementation of saturation effects in the description of the scattering process $[9,10]$. In fact, in the small- $x$ range, many experimental data with a number of features are successfully described by the dipole model. By requiring higher-order corrections to the Balitsky-Kovchegov-JIMWLK (BK-JIMWLK) equations $[3-8,11-13]$, an important progress was achieved in the past years. Recently, through the calculation of higher-order corrections to the BK-JIMWLK equations, an important step towards promoting the CGC to a practical phenomenological tool was performed [14-17]. In particular, the calculation of running coupling $\left(\alpha_{s}\right)$ corrections to the evolution kernel of the Balitsky-Kovchegov (BK) equation [18, 19] renders the possibility to describe various data at high energies. In particular, the BK equation with running coupling (referred to as rcBK equation $[18,19]$ ) grasps most of the higher-order effects and incorporates nonlinear high gluon density effects. These processes close the gap between the first principle theory calculations and the experimental data.

In the CGC formalism, by using varying degrees of approximations and models, many researchers investigated single inclusive hadron productions in proton-nucleus $(p A)$ collisions at the Relativistic Heavy Ion Collider (RHIC) and the Large Hadron Collider (LHC) [20-31]. The CGC framework describes well single-particle distributions in both 
proton-proton $(p p)$ and deuton-gold $(d+A u)$ collisions, as well as the depletion of nuclear modification factors $R_{d \mathrm{Au}}$ $[27,29]$. In deuton-nucleus $(d A)$ collisions at the RHIC, the observed suppression of single inclusive hadron production and the disappearance of the away-side peak in doublehadron production in the forward rapidity region are perhaps the strongest evidence for the importance and possibly dominance of saturation effects. The CGC calculations firstly predicted these effects [32] and later on quantitatively accounted for such suppression $[33,34]$. This will be tested further at the LHC where, due to the larger energy of the collisions, the CGC dynamics in a much larger kinematic region can be probed. In the theoretical framework of the CGC, the single inclusive hadron production cross-section captures the saturation dynamics. The most important ingredient of the cross-section is the fundamental (or adjoint) dipole crosssection, that is, the imaginary part of the quark-antiquark $(q \bar{q})$ scattering amplitude on a proton or nucleus target. This dipole cross-section satisfies the BK-JIMWLK evolution equations $[3-8,11-13]$. It also resums the small- $x$ and high gluon density effects. With next-to-leading-order (NLO) accuracy $[14-17,35]$, one now knows the evolution equation for the dipole cross-section.

Because of the larger valence charge density in a nucleus, the CGC effect in a nucleus is expected to be more enhanced than that in a proton. However, due to a large amount of highquality DIS data on protons at small- $x$, the most exhaustive searches of the saturation phenomenon are performed using data on proton reactions. The global fits performed by the Albacete-Armesto-Milhano-Quiroga-Salgado (AAMQS) group [36-38], in ep collision at the HERA [39], show that the rcBK equation successfully accounts for the $x$-dependence of inclusive structure functions at small- $x$. We would like to point out that the AAMQS initial condition is not derived from an effective action (Gaussian action) for the large- $x$ valence charges. Based on a non-Gaussian action (quartic action), which involves the subleading correction of valence color charges, Dumitru and Petreska derived a new dipole scattering amplitude [40]. Thus, we may compare the two different initial conditions (AAMQS and quartic action), which could illustrate the effect of valence color charges in $p A$ collisions at high energy. In particular, as the number of nucleons is finite, the process of scattering then involves the correction of valence color charges effects.

Due to the scientific significances and interests, in this paper, we study the inclusive particle productions in $p p$ and $d+\mathrm{Au}$ collisions at the RHIC energy in the framework of the CGC theory by using the previously mentioned two initial conditions. Then, the inclusive particle productions in $p A$ collisions at the LHC energies are predicted by us. As we know, the situations at central rapidity and forward rapidity are different. To focus our attention on fragmentation region, where the leading nucleons and cold spectators are affected by each other, we study only the inclusive particle productions at forward rapidity in this paper. In Sections 2 and 3, we introduce and review the high-energy evolution equation and give single inclusive hadron production, respectively. The conclusions are given in Section 4.

\section{High-Energy Evolution Equation}

2.1. The BK Equation with Running Coupling. In the CGC framework, one needs the BK-JIMWLK equations to describe the quantum evolution of hadron structure towards small- $x$. The BK-JIMWLK equations are equivalent to an infinite set of coupled nonlinear equations for the different Wilson line correlators, where these correlators systematically incorporate small- $x$ gluon emission to all orders [3-8]. In the large number of colors (large- $N_{c}$ ) limit, the JIMWLK evolution equations are reduced to the BK equation [11-13], while the later one is a closed-form equation for the evolution of the dipole amplitude.

According to $[11-14,18]$, the rcBK equation can be given in the following form:

$$
\begin{aligned}
& \frac{\partial \mathcal{N}_{F}(r, x)}{\partial \ln \left(x_{0} / x\right)} \\
& \quad=\int d^{2} \vec{r}_{1} K^{\mathrm{run}}\left(\vec{r}, \vec{r}_{1}, \vec{r}_{2}\right) \\
& \quad \times\left[\mathcal{N}_{F}\left(r_{1}, x\right)+\mathcal{N}_{F}\left(r_{2}, x\right)\right. \\
& \left.\quad-\mathcal{N}_{F}(r, x)-\mathcal{N}_{F}\left(r_{1}, x\right) \mathscr{N}_{F}\left(r_{2}, x\right)\right],
\end{aligned}
$$

where $\mathcal{N}_{F}, x_{0}$, and $\vec{r}\left(=\vec{r}_{1}+\vec{r}_{2}\right)$ denote the forward dipoletarget scattering amplitude in the fundamental $(F)$ representation, the value of $x$ where the evolution starts, and the transverse dipole size, respectively. In order to give some numerical solutions and related applications of (1), we can use a kernel validity to running coupling accuracy. According to Balitsky's prescription [14], the kernel $K^{\text {run }}$ in (1) can be presented as follows:

$$
\begin{aligned}
& K^{\text {run }}\left(\vec{r}, \vec{r}_{1}, \vec{r}_{2}\right) \\
& =\frac{N_{c} \alpha_{s}\left(r^{2}\right)}{2 \pi^{2}}\left[\frac{1}{r_{1}^{2}}\left(\frac{\alpha_{s}\left(r_{1}^{2}\right)}{\alpha_{s}\left(r_{2}^{2}\right)}-1\right)\right. \\
& \\
& \left.\quad+\frac{r^{2}}{r_{1}^{2} r_{2}^{2}}+\frac{1}{r_{2}^{2}}\left(\frac{\alpha_{s}\left(r_{2}^{2}\right)}{\alpha_{s}\left(r_{1}^{2}\right)}-1\right)\right] .
\end{aligned}
$$

For the running coupling in the above equation, we may use the scheme proposed in [37] at one-loop level.

In particular, in the large- $N_{c}$ limit, one has the following relation between the adjoint $(A)$ and fundamental dipoles:

$$
\mathcal{N}_{A}(r, Y)=2 \mathcal{N}_{F}(r, Y)-\mathcal{N}_{F}^{2}(r, Y) .
$$

The amplitude $\mathcal{N}_{A(F)}$ incorporates all multiscattering between a projectile color dipole and the target and encodes the small- $x$ dynamics. In the CGC framework, it can be obtained from the solution of rcBK evolution with a given initial condition.

2.2. Initial Condition: Beyond the McLerran-Venugopalan Model. The initial conditions required by the high-energy evolution equations are at a rapidity of $Y=\ln \left(x_{0} / x\right)=0$. Generally, one takes $x_{0}=10^{-2}$. The dynamical equations 
were derived by McLerran and Venugopalan $[41,42]$ in the limit of an infinitely large nucleus. Let $\rho^{a}\left(x_{\perp}, x^{-}\right)$denote the classical color charge density per unit transverse area $x_{\perp}$ and longitudinal length $x^{-}$, where kinetic terms $\rho$ are neglected because the transverse momenta are assumed to be small. In the McLerran-Venugopalan (MV) model [41, $42]$, the large- $x$ valence color charges are then described as recoilless sources on the light cone with $\rho^{a}\left(x_{\perp}, x^{-}\right)$. As $A \rightarrow$ $\infty$, the variance of the valence color charge density $\sim A^{1 / 3}$ grows large corresponding to a very high-density $\rho$ of charges. According to the central limit theorem, the fluctuations of the color charges are described by a Gaussian effective action. Generally, the high multiplicity $p p$ collision corresponds to an unusually high valence color charge density [43]. Then, in this case, we can use the Gaussian effective action to describe it. However, for the real case with the mass number $A$, the number of valence color charges is finite. Particularly in $p p$ and peripheral nucleus-nucleus $(A A)$ collisions, the considered number is very limited. Therefore, one needs to consider extensions of the MV model action to involve higher powers of the color charge density.

In fact, Jeon and Venugopalan $[44,45]$ introduced an "odderon" operator contribution $-d^{a b c} \rho^{a} \rho^{b} \rho^{c} / \kappa_{3}$ to the effective action with $\kappa_{3} \sim g^{3}\left(A / \pi R^{2}\right)^{2}$, where $R$ denotes the radius of the nucleus. Dumitru et al. [46] introduced the quartic order contribution $\delta^{a b} \delta^{c d} \rho^{a} \rho^{b} \rho^{c} \rho^{d} / \kappa_{4}$ with $\kappa_{4} \sim$ $g^{4}\left(A / \pi R^{2}\right)^{3}$. Finally, to go beyond the limit of infinite valence charge density, the effective action is written as follows [46]:

$$
\begin{aligned}
& S[\rho(x)] \\
& \simeq \int d^{2} x\left[\frac{\delta^{a b} \rho^{a} \rho^{b}}{2 \mu^{2}}-\frac{d^{a b c} \rho^{a} \rho^{b} \rho^{c}}{\kappa_{3}}\right. \\
& \left.+\frac{\delta^{a b} \delta^{c d}+\delta^{a c} \delta^{b d}+\delta^{a d} \delta^{b c}}{\kappa_{4}} \rho^{a} \rho^{b} \rho^{c} \rho^{d}\right] .
\end{aligned}
$$

Based on the new effective action, including the first subleading correction in the semiclassical approximation, Dumitru and Petreska [40] introduced the dipole scattering amplitude $\mathcal{N}(r)$ on a dense target. This dipole scattering amplitude can be matched with a phenomenological proton fit by Albacete et al. $[36,37]$ over a broad range of dipole size $r$ and can provide a definite prediction for the $A$-dependence for heavy-ion targets. According to [40], this dipole scattering amplitude has the following form:

$$
\mathcal{N}(r)=\frac{Q_{s}^{2} r^{2}}{4} \ln \left(\frac{1}{r \Lambda}\right)-\beta Q_{s}^{2} r^{2} \ln ^{3}\left(\frac{1}{r \Lambda}\right), \quad\left(r^{2} Q_{s}^{2}<1\right),
$$

where $Q_{s}$ and $\Lambda$ denote the initial saturation momentum scale and the infrared scale, respectively, and

$$
\beta \equiv \frac{C_{F}^{2}}{6 \pi^{3}} \frac{g^{8}}{Q_{s}^{2} \kappa_{4}}\left[\int_{-\infty}^{\infty} d z^{-} \mu^{4}\left(z^{-}\right)\right]^{2} \simeq \frac{1}{100}, \quad(A=1) .
$$

For the nucleus with a mass $A$, we have $\beta_{A} \sim A^{-2 / 3}$.
As discussed in [40], the amplitude decreases sharply at very short distances. This phenomenon may not be reasonable. As we note, that the new term of the valence color charge has an extremely larger contribution than the other one at very small dipole size. This term corresponds to strong interaction caused by higher-order correlations of valence color charges. More carefully, we have $\mathcal{N}(r) \sim r^{2}$ in the limit of $r \rightarrow 0$. Based on this behavior, the valence color charge term is assumed to be controlled by a weight function $\gamma_{2}(r)$ to go smoothly to the scattering amplitude. The first term is assumed to be controlled by a weight function $\gamma_{1}(r)$. The new scattering amplitude is given by the following:

$$
\begin{aligned}
\mathcal{N}(r)= & \gamma_{1}(r) \frac{Q_{s}^{2} r^{2}}{4} \ln \left(\frac{1}{r \Lambda}\right) \\
& -\gamma_{2}(r) \beta Q_{s}^{2} r^{2} \ln ^{3}\left(\frac{1}{r \Lambda}\right), \quad\left(r^{2} Q_{s}^{2}<1\right) .
\end{aligned}
$$

Following this procedure, the unreasonable phenomena can be avoided. It should be noted that taking the weight function involved is just an experiential treatment. We also assume experientially that the weight functions have the following form:

$$
\begin{gathered}
\gamma_{1}(r)=0.001+0.999 \exp \left(-\frac{0.0002}{r^{2} Q_{s}^{2}}\right), \\
\gamma_{2}(r)=\exp \left(-\frac{0.00021}{r^{2} Q_{s}^{2}}\right) .
\end{gathered}
$$

The parameters above are obtained by balanceing the two terms in the amplitude. That means the contribution of the new term is vanished while the first term is domainal at very short distance. Then, the scattering amplitude for a dipole in the adjoint representation with a given $\beta$ can be obtained. We would like to point out that to regularize the behavior at large $r$ in the calculation, we have replaced $\ln (1 / r \Lambda)$ by $\ln (e+(1 / r \Lambda))$ in $(7)$ and assumed an exponentiation of the $\mathcal{O}\left(r^{2}\right)$ expression. This does not affect the behavior at $r Q_{s} \leq 1$.

The dipole scattering amplitude for a proton target was fitted by the AAMQS model [36-38] which gives the amplitude, in the initial condition for a small- $x$ evolution, to be

$$
\begin{aligned}
& \mathcal{N}_{\text {AAMQS }}\left(r, x_{0}=0.01\right) \\
& \quad=1-\exp \left[-\frac{1}{4}\left(r^{2} Q_{s}^{2}\left(x_{0}\right)\right)^{\gamma} \ln \left(e+\frac{1}{r \Lambda}\right)\right],
\end{aligned}
$$

where the infrared scale $\Lambda$ is taken to be $0.241 \mathrm{GeV}$. We notice that the case with $\gamma=1$ corresponds to the MV model which was derived from an effective action (Gaussian action) for the large- $x$ valence charges. The free parameters are parameter $\gamma$ and the initial saturation scale $Q_{s}$, which is probed by quarks. For the proton and nuclear targets, we use respectively, the notations $Q_{0 p}$ and $Q_{0 A}$ for clearness. From a global fit to proton structure functions in DIS in the small- $x$ region and single inclusive hadron data in $p p$ collisions at the RHIC and the LHC, the proton initial saturation scale gives $Q_{0 p}^{2} \simeq 0.168 \mathrm{GeV}^{2}$ and $\gamma \simeq 1.119$. Simultaneously, this model provides a good description of charged hadron transverse 


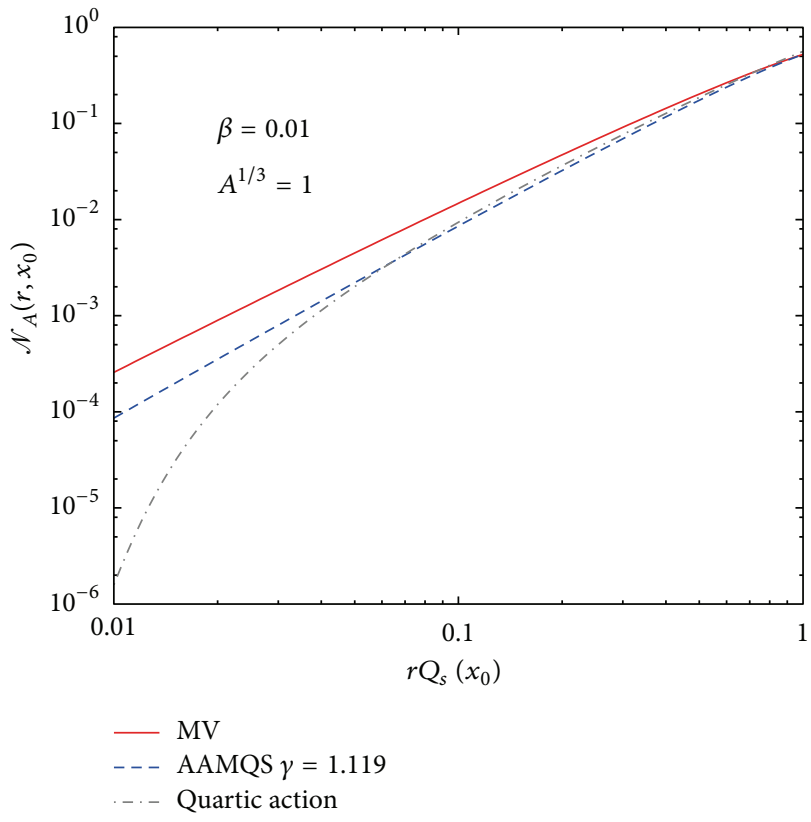

(a)

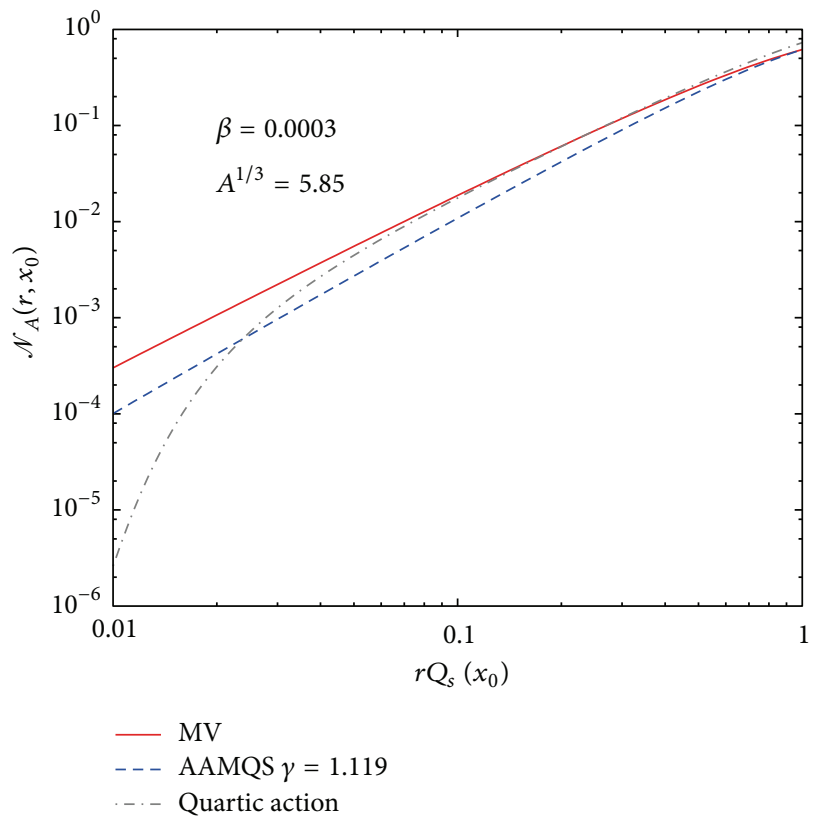

(b)

Figure 1: (a) Dependence of dipole scattering amplitude in an adjoint representation $\left(\mathcal{N}_{A}=2 \mathcal{N}_{F}-\mathscr{N}_{F}^{2}\right)$ on $r Q_{s}\left(x_{0}\right)$ with three different models indicated in the figure. (a) is for a proton target assuming $Q_{s}^{2}=0.168 \mathrm{GeV}^{2}$ and $\Lambda^{2}=0.058 \mathrm{GeV}^{2}$. (b) is for a nucleus target with $A=200$ (which is between $\mathrm{Au}$ and $\mathrm{Pb}$ ), $\mathrm{Q}_{s}^{2} \sim A^{1 / 3}$, and $\beta_{A} \sim A^{-2 / 3}$ (or $\beta=0.0003$ ).

momentum distributions in $p p$ collision at the center-ofmass energy per nucleon pair $\sqrt{s_{N N}}=7 \mathrm{TeV}[47,48]$.

Solving the rcBK equation related to the impact-parameter is still an open question. This renders that in (1) the impact parameter dependence of the collisions is ignored. In fact, this question may not be important for the minimumbias analysis because the initial saturation scale $Q_{0 A}$ of a nucleus can be considered as an averaged value and extracted from the minimum-bias data. In the minimum-bias collisions, one may assume that $Q_{0 A}^{2}=C A^{1 / 3} Q_{0 p}^{2}$, where the parameter $C$ is fixed from a fit to data. In [49], it was shown that $C \approx 0.5$ if the DIS data is used for the description of heavy nuclear targets. This result is consistent with the inclusive hadron production data in minimum-bias $d+\mathrm{Au}$ collisions at the RHIC energy, which gives an initial saturation scale within $Q_{0 A}^{2} \approx 3 \div 4 Q_{0 p}^{2}$. The uncertainties associated to the variation of initial saturation scale in the rcBK evolution equation should be taken into account.

The dependence of dipole scattering amplitudes in the adjoint representation $\left(\mathcal{N}_{A}=2 \mathcal{N}_{F}-\mathcal{N}_{F}^{2}\right)$ on $r Q_{s}\left(x_{0}\right)$ are shown in Figure 1 with three different models: MV (the solid curves), AAMQS with $\gamma=1.119$ (the dashed curves), and quartic action (the dashed-dotted curves). Figure 1(a) is for a proton assuming that $Q_{s}^{2}=0.168 \mathrm{GeV}^{2}, \Lambda^{2}=0.058 \mathrm{GeV}^{2}$, and $\beta=0.01$. Figure $1(\mathrm{~b})$ is for a nucleus with $A=200$ (which is between $\mathrm{Au}$ and $\mathrm{Pb}$ ), $\mathrm{Q}_{s}^{2} \sim A^{1 / 3}$, and $\beta_{A} \sim$ $A^{-2 / 3}$ (or $\beta=0.0003$ ). One can see that both the dipole scattering amplitudes resulted from the quartic action and AAMQS models are beyond the MV model. The result of the quartic action is similar to the AAMQS model over a broad $r Q_{s}\left(x_{0}\right)$ range. At a small size of dipole, the result of the quartic action is much smaller than that of AAMQS. Dumitru and Petreska [40] argued that the suppression of the bremsstrahlung tails by the subleading term possibly explains the difference between the AAMQS and MV models within relevant region $0.05<r Q_{s}<1$. At very small $r$, however, the subleading correction starts to dominate the leading one. This implies the breakdown of the simple perturbative expansion.

To understand more easily the above phenomenon, the scattering amplitude $\mathcal{N}(r, y)$ for a dipole in coordinate space at different rapidities is given in Figure 2. The solid and dashed-dotted curves represent the results of the AAMQS with $\gamma=1.119$ and quartic action, respectively. From the right curve to the left one, the corresponding rapidities are $y=0,5$, and 10 , respectively. One can see that, at $y=0$, there is a very little difference between the results obtained using the two different initial conditions. At $y=5$ and 10, the two types of results are almost the same. These phenomena are caused by the gluon distribution of the proton itself, which is independent of any particular reactions and renders nearly the same result.

\subsection{Structure Function and Comparison with Experimental} Data. To check the validity of these calculations, let us calculate the proton structure function $F_{2}$ at small- $x$. At $x \ll$ 1 , the inclusive structure function obtained from DIS process is given by [50]

$$
F_{2}\left(x, Q^{2}\right)=\frac{Q^{2}}{4 \pi^{2} \alpha_{\mathrm{em}}}\left(\sigma_{T}^{\gamma^{*} p}+\sigma_{L}^{\gamma^{*} p}\right),
$$




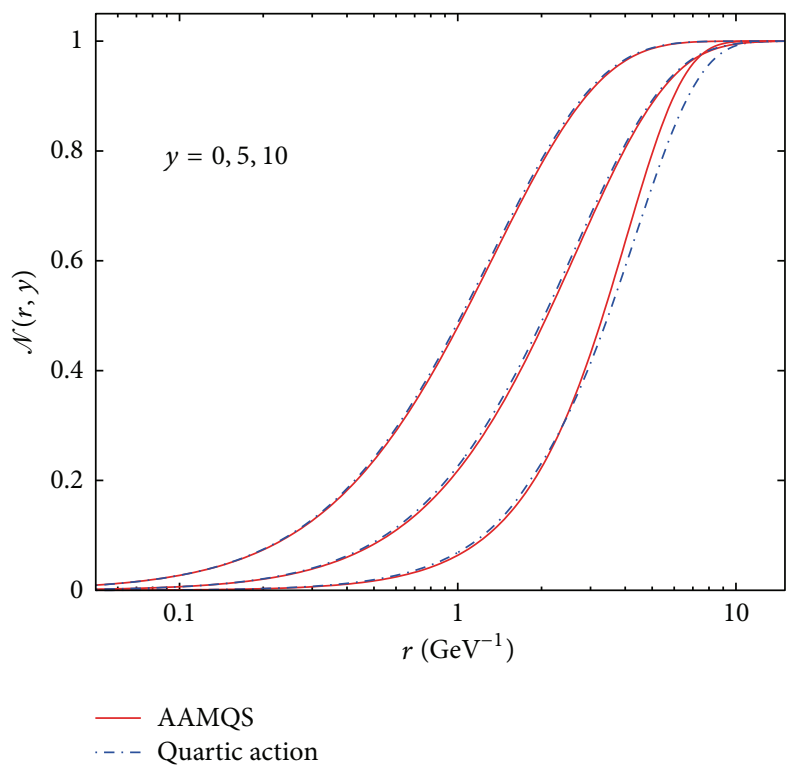

FIGURE 2: Dipole scattering amplitude $\mathcal{N}(r, y)$ in coordinate space at different rapidities with two different initial conditions indicated in the figure. From the right curve to the left one, the corresponding rapidities are $y=0,5$, and 10 , respectively.

where $\alpha_{\mathrm{em}}, Q^{2}$, and $\sigma_{T, L}^{\gamma^{*} p}$ denote the electromagnetic coupling, the transverse resolution scale, and the virtual photonproton $\left(\gamma^{*} p\right)$ cross-section for transverse $(T)$ and longitudinal $(L)$ polarization states of the virtual photon, respectively. In the dipole model, the total $\gamma^{*} p$ cross-section is given by

$$
\sigma_{T, L}^{\gamma^{*} p}=\sum_{f} \int d^{2} r_{T} \int_{0}^{1} d z\left[\Psi^{*} \Psi\right]_{T, L}^{f}\left(z, Q^{2}\right) \sigma_{q \bar{q}}\left(x, r_{T}\right)
$$

where $z, r_{T}, \Psi, f$, and $\sigma_{q \bar{q}}\left(x, r_{T}\right)$ denote the fraction of longitudinal momentum of the virtual photon carried by the quark, the transverse separation between the quark and the antiquark, the light-cone wavefunction for the photon splitting into a $q \bar{q}$ pair $[51,52]$, the flavor of the quark, and the dipole-proton cross-section, respectively. By using the optical theorem, $\sigma_{q \bar{q}}\left(x, r_{T}\right)$ can be obtained from the elastic dipoleproton scattering amplitude $\mathscr{A}\left(x, r_{T}, \Delta\right)$,

$$
\begin{aligned}
\sigma_{q \bar{q}}\left(x, r_{T}\right) & =2 \operatorname{Im} \mathscr{A}\left(x, r_{T}, \Delta\right) \\
& =2 \int d^{2} b_{T} \mathcal{N}\left(x, r_{T}, b_{T}\right)=\sigma_{0} \mathcal{N}\left(x, r_{T}\right),
\end{aligned}
$$

where $\mathcal{N}\left(x, r_{T}, b_{T}\right), b_{T}$, and $\sigma_{0}$ denote the imaginary part of the forward elastic dipole-proton scattering amplitude, the impact parameter of the dipole-target collisions, and the normalization constant obtained by the $b_{T}$ integration, respectively.

To see the dependence of structure function $F_{2}$ on $x$, in Figure 3 we show the comparisons between the experimental data (squares) in ep collision [53-55] and the results by solving rcBK equation with the two different initial conditions, AAMQS (the solid curves) and quartic action (the dasheddotted curves), by using (2) and (3). Figures 3(a), 3(b), 3(c), and $3(\mathrm{~d})$ are the results for different values of $Q^{2}$. In the calculation, we take $\sigma_{0}=30.265 \mathrm{mb}, Q_{0 s}^{2}=0.168 \mathrm{GeV}^{2}$, $C=1.715$, and $\alpha_{f r}=1$ in the running coupling in (2) for the four $Q^{2}$ values in Figure 4. One can see that the two different initial conditions describe well the $F_{2}$ data, although their behaviors in Figure 1 on $\mathcal{N}_{A}\left(r, x_{0}\right)$ at small $r Q_{s}\left(x_{0}\right)$ are different.

\section{Single Inclusive Hadron Production}

3.1. Hybrid Formalisms. In asymmetric collisions such as $p A$ to hadron $(h)$ and others $(X)$ at high energy, the scattering occurs between a dilute system of parton and a dense one of nucleus $[56,57]$. The cross section for single inclusive hadron production at forward rapidity is given by [58]

$$
\begin{aligned}
& \frac{d N^{p A \rightarrow h X}}{d^{2} p_{T} d \eta} \\
& =\frac{K}{(2 \pi)^{2}}\left[\int_{x_{F}}^{1} \frac{d z}{z^{2}}\right. \\
& \times\left[x_{1} f_{g}\left(x_{1}, Q^{2}\right) N_{A}\left(x_{2}, \frac{p_{T}}{z}\right) D_{h / g}(z, Q)\right. \\
& +\Sigma_{q} x_{1} f_{q}\left(x_{1}, Q^{2}\right) \\
& \left.\left.\quad \times N_{x_{F}}^{1} \frac{d z}{z^{2}} \frac{\alpha_{s}}{2 \pi^{2}} \frac{z^{4}}{p_{T}^{4}} \frac{p_{T}}{z}\right) D_{h / q}(z, Q)\right] \\
& \times \int_{k_{T}^{2}<Q^{2}} d^{2} k_{T} k_{T}^{2} N_{F}\left(k_{T}, x_{2}\right)
\end{aligned}
$$




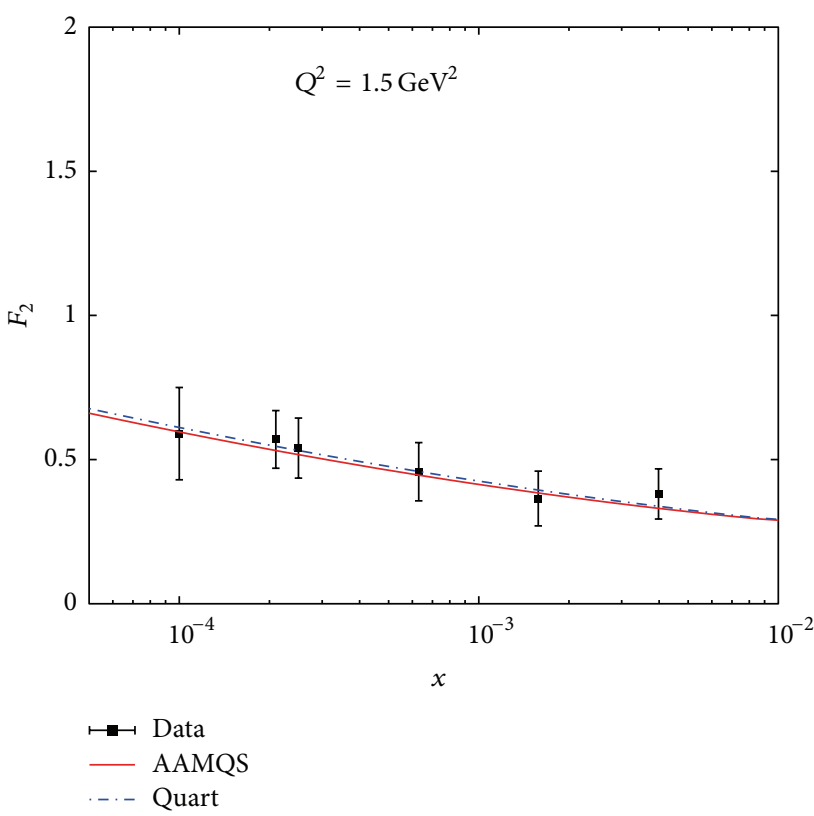

(a)

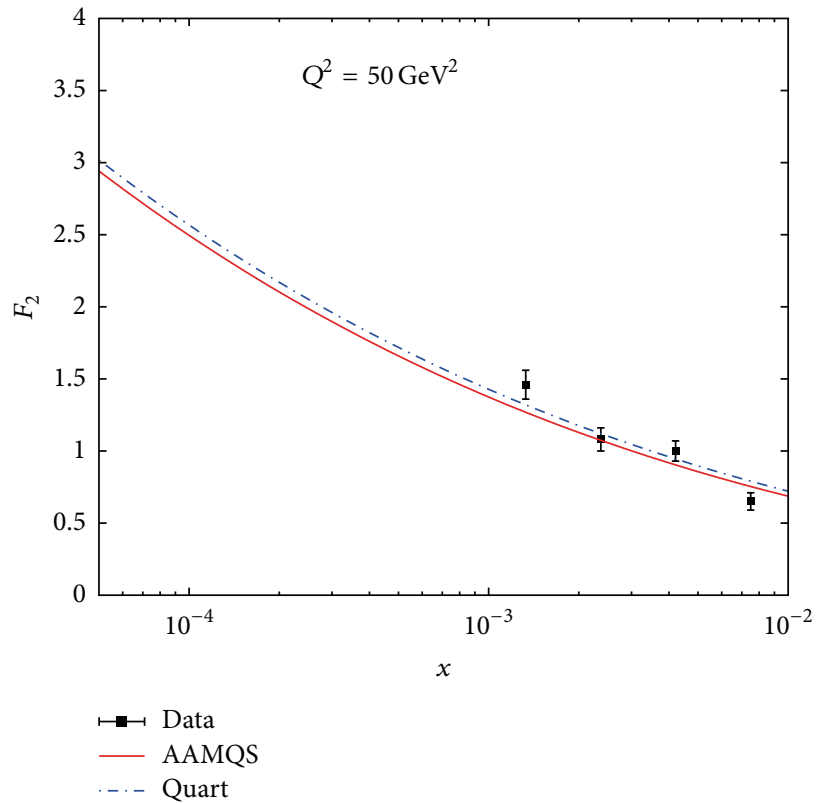

(c)

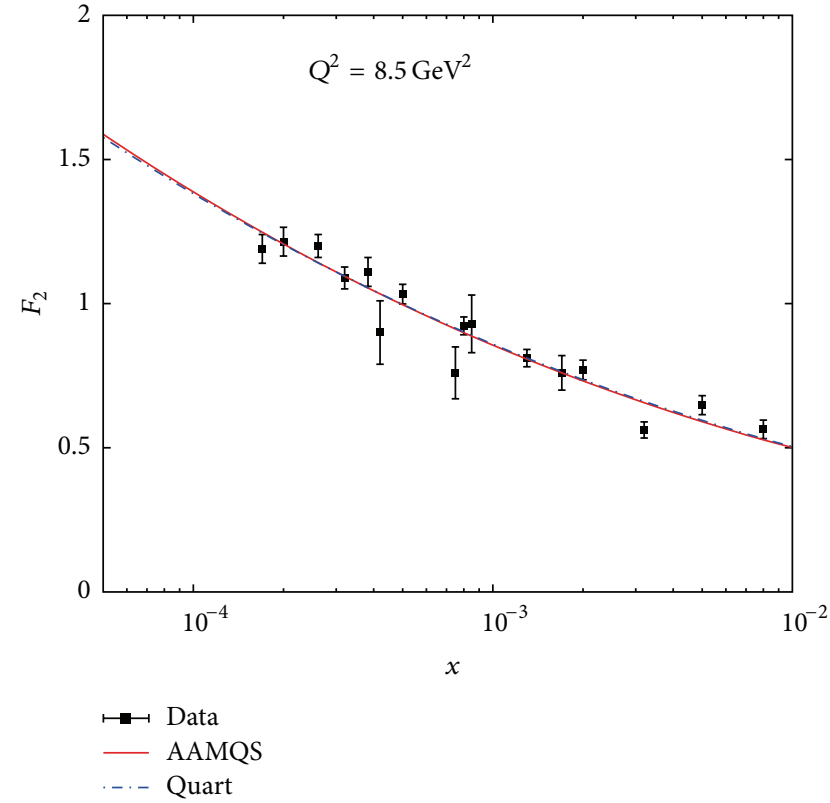

(b)

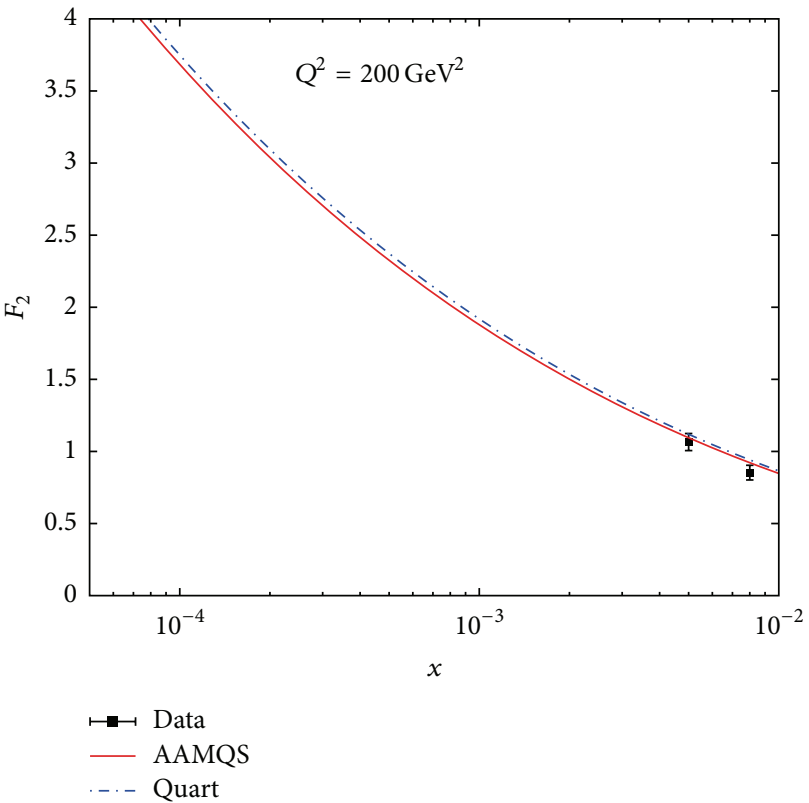

(d)

FIgURE 3: Proton structure function $F_{2}\left(x, Q^{2}\right)$ versus $x$ at different $Q^{2}$. The squares represent the experimental data of the HERA Collaboration [53-55]. The solid and dashed-dotted curves represent the results calculated by the AAMQS and quartic action initial conditions, respectively.

$$
\begin{aligned}
& \times \int_{x_{1}}^{1} \frac{d \xi}{\xi} \\
& \times \sum_{i, j=q, \bar{q}, g} w_{i / j}(\xi) P_{i / j}(\xi) x_{1} \\
&\left.\quad \times f_{j}\left(\frac{x_{1}}{\xi}, Q\right) D_{h / i}(z, Q)\right],
\end{aligned}
$$

where the quantities $K, \xi, k_{T}$, $\eta$, and $p_{T}$ denote the normalization constant, the splitting fraction for a given parton splitting into two others, the small transverse momentum which is strongly affected by target saturation effects, the pseudorapidity of the produced hadron, and the transverse momentum of the considered hadron, respectively; the functions $f_{i}\left(x, Q^{2}\right), D_{h / i}(z, Q), w_{i / j}(\xi)$, and $P_{i / j}(\xi)$ are the parton distribution functions of the incoming proton which depends on the light-cone momentum fraction $x$ and the hard scale $Q$, the hadron fragmentation function of the $i$ th parton to 


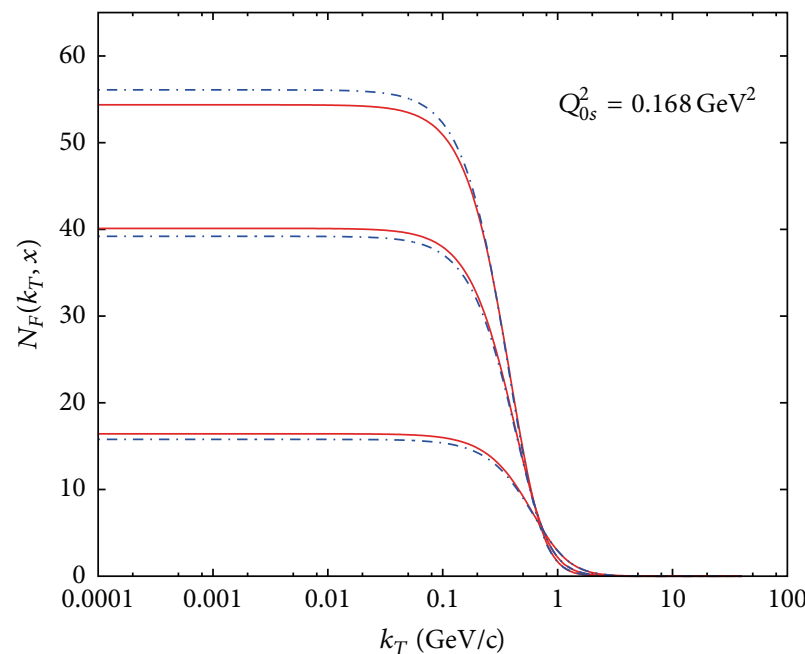

- AAMQS

... Quartic action

(a)

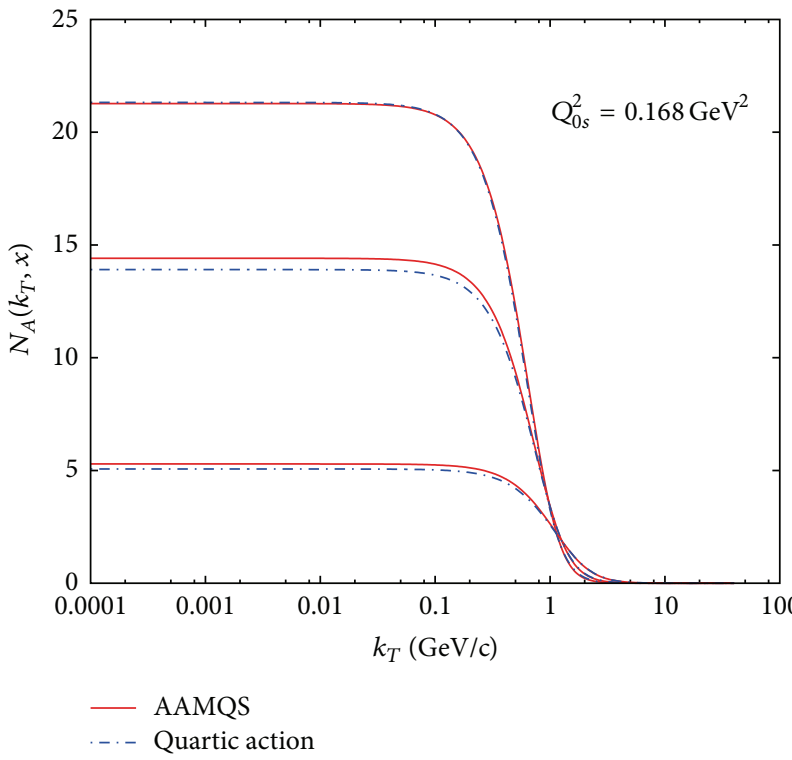

(c)

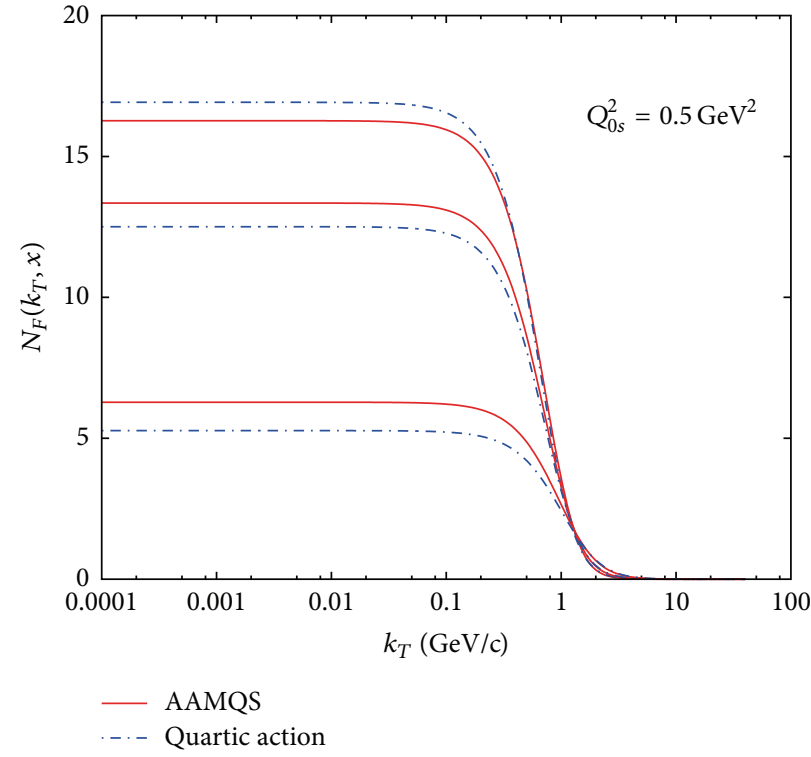

(b)

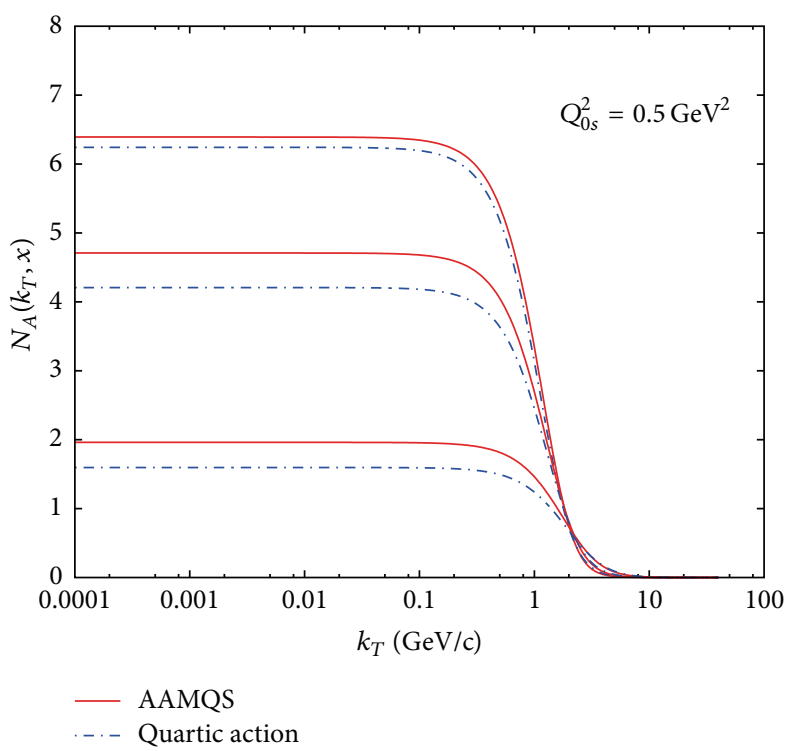

(d)

FIGURE 4: Dependence of the dipole scattering amplitude $N_{A(F)}\left(k_{T}, x\right)$, the Fourier transform of $1-\mathcal{N}_{A(F)}(r, Y)(15)$, on $k_{T}$ for (a) a proton target and (b) a nucleus target with $A=200$ at different rapidities with two different initial conditions indicated in the figure. From the upper curve to the lowest one, the corresponding rapidities are $y=2,4$, and 8 , respectively.

the final hadron $h$ with a momentum fraction $z$, the inelastic weight function, and the Altarelli and Parisi [59] splitting function, respectively. The last two functions can be found in equations (74-77) of [58] and equations (16.26-16.29) of [60] for more details. The longitudinal momentum fractions $x_{1}$ and $x_{2}$ are defined as follows:

$$
\begin{gathered}
x_{1}=\frac{x_{F}}{z} \approx \frac{p_{T}}{z \sqrt{s_{N N}}} e^{\eta}, \\
x_{2}=x_{1} e^{-2 \eta} \approx \frac{p_{T}}{z \sqrt{s_{N N}}} e^{-\eta},
\end{gathered}
$$

where $x_{F}$ is the Feynman variable in another form. In the above calculation, the hadron masses are neglected since we are only interested in the light hadron production at high $p_{T}$, which results the rapidity to equal approximately to the pseudorapidity. As in the two-dimensional Fourier transform, the amplitude $N_{A}\left(N_{F}\right)$ in (13) can be given by

$$
N_{A(F)}\left(k_{T}, x\right)=\int d^{2} \vec{r} e^{-i \vec{k}_{T} \cdot \vec{r}}\left(1-\mathcal{N}_{A(F)}\left(r, Y=\ln \left(\frac{x_{0}}{x}\right)\right)\right) .
$$




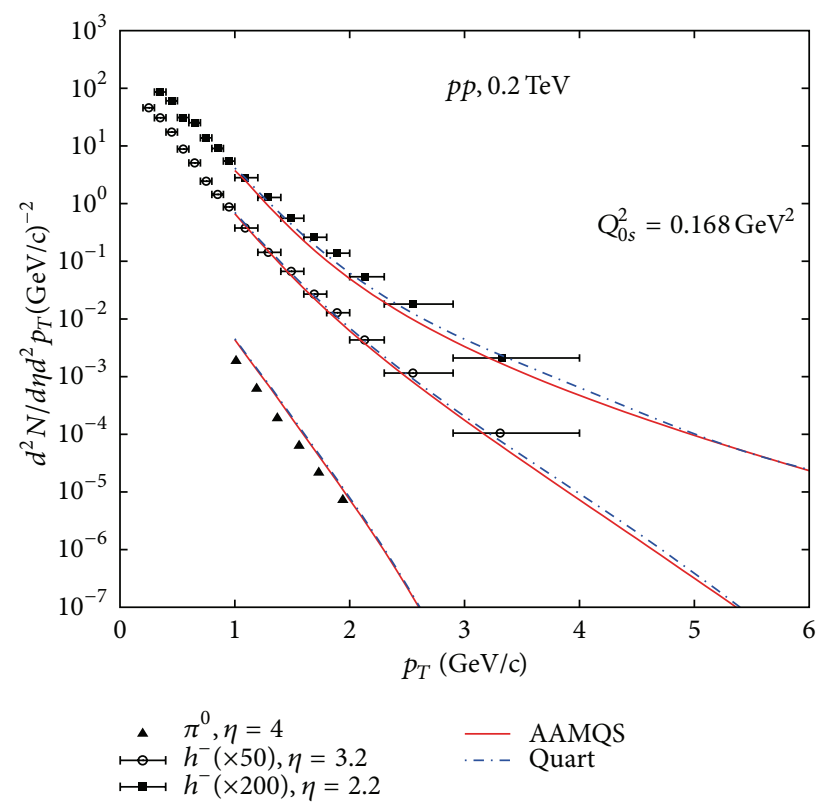

(a)

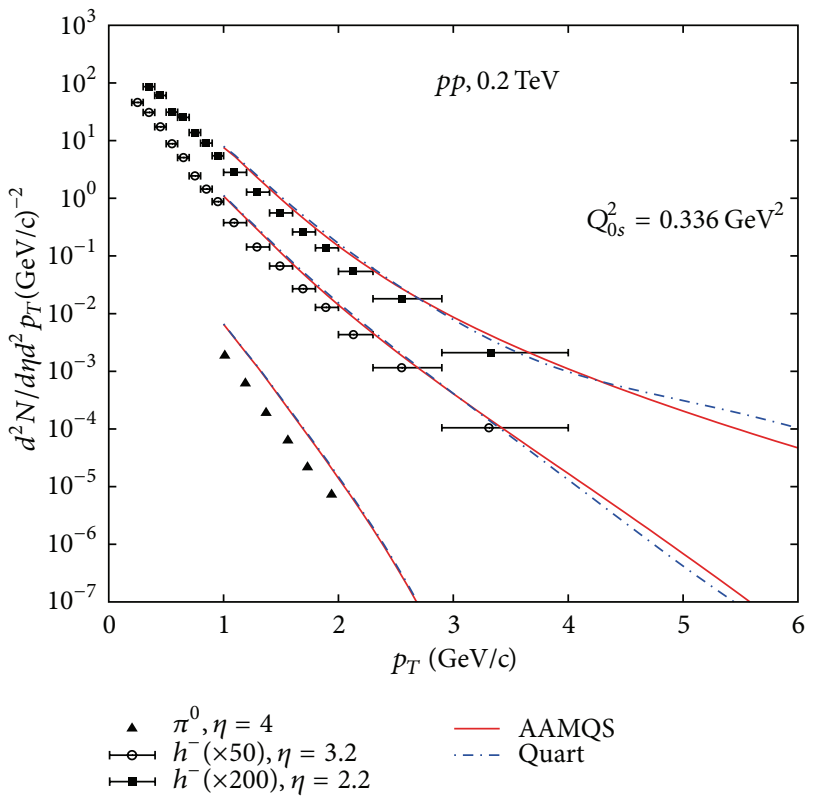

(b)

FIGURE 5: Neutral pion and negatively charged hadron yields in $p p$ collisions at $\sqrt{s_{N N}}=0.2 \mathrm{TeV}$. The symbols represent the experimental data at different pseudorapidities measured by the STAR [56] and BRAHMS Collaborations [57]. The solid and dashed-dotted curves represent the results calculated by the AAMQS and quartic action, respectively, with $Q_{0 s}^{2}=0.168 \mathrm{GeV}^{2}$ (a) and $0.336 \mathrm{GeV}^{2}$ (b) in the calculations.

The first integral in (13) denotes the elastic term which was derived in [23] and corresponds to the scattering of collinear partons from the projectile on the target. In the process corresponding to the elastic term, a quark from the projectile scatters on the target and radiates a gluon either before or after the scattering. The incoming quark, the outgoing quark, and the radiated gluon can all multiply scatters on the target. Because the incoming parton initially has zero transverse momentum, but picks up transverse momentum of order $Q_{s}$ after multiply scattering on the target, the elastic term is the most important one. The other part in (13) denotes the inelastic term which was derived in [58] and is important only when the produced hadron has a much higher transverse momentum than $Q_{s}$. The inelastic term corresponds to high transverse momentum partons radiated from the incoming partons in the projectile wave function. The partons with high transverse momentum may scatter off the target with only a small momentum transfer, and finally they are fragmentated into high- $p_{T}$ hadrons.

We show the dipole scattering amplitude $N_{A(F)}\left(k_{T}, x\right)$ and the Fourier transform of $1-\mathcal{N}_{A(F)}(r, Y)(15)$, for a proton target and a nucleus target with $A=200$ in Figures 4(a) and 4(c), as well as Figures 4(b) and 4(d), respectively. The solid and dashed-dotted curves represents the results of the AAMQS with $\gamma=1.119$ and quartic action, respectively. In Figures $4(\mathrm{a})$ and $4(\mathrm{c})$, we take $Q_{0 s}^{2}=0.168 \mathrm{GeV}^{2}$; in Figures $4(\mathrm{~b})$ and $4(\mathrm{~d})$, we take $Q_{0 s}^{2}=0.5 \mathrm{GeV}^{2}$. From the top curve to the lowest one, the corresponding rapidities are $y=2,4$, and 8 , respectively. One can see that in most of the cases the differences between the two types of results obtained using the two initial conditions are small. The value of $N_{A(F)}\left(k_{T}, x\right)$ for a proton target is much larger than that of $N_{A(F)}\left(k_{T}, x\right) / A^{1 / 3}$ for a nucleus with $A=200$.

3.2. Numerical Results and Predictions. To give the numerical results of the hybrid formula, we can use the CT10 NLO [61] parton distribution functions and the DSS NLO [56, 62] fragmentation functions. The only input for the rcBK equation is the initial conditions for the evolution of the dipole amplitude. Our numerical results will be given by using the AAMQS and the quartic action, respectively. As already mentioned previously, the inelastic term in (13) involves an additional coupling factor $\alpha_{s}$ at NLO or named a running coupling scale which is considered as a free parameter in [57]. In this work, we take $\alpha_{s}=0.1$ which is the same as that in [57].

Our aim is not to fit the experimental data but to use the CGC theoretical tools available in low- $x$ physics to highlight the uncertainties involved in making robust predictions for the upcoming $p A$ collisions at the LHC. In all the calculations, we take $K=1$ in (13). However, the STAR data at very forward pseudorapidity $(\eta=4)$ shows that a $K$-factor of $\sim 0.4 \div 0.6$ may be needed (see [27] for more details). The necessity of such a small value of $K$-factor may correspond to a large$x$ phenomena, which is not included in the CGC at very forward (pseudo)rapidity region.

In Figure 5 we show the single inclusive hadron production yields (transverse momentum distributions) in $\sqrt{s_{N N}}=$ $0.2 \mathrm{TeV} p p$ collisions at different pseudorapidities using the rcBK dipole solution with two different initial conditions, AAMQS (the solid curves) and quartic action (the dasheddotted curves). The symbols (triangles, circles, and squares) 


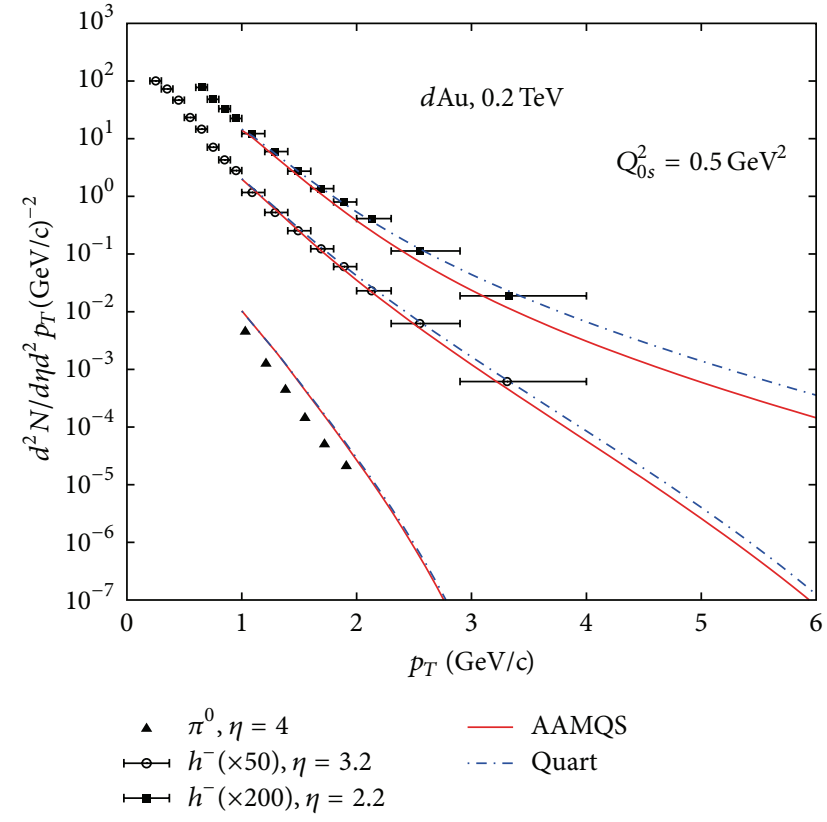

(a)

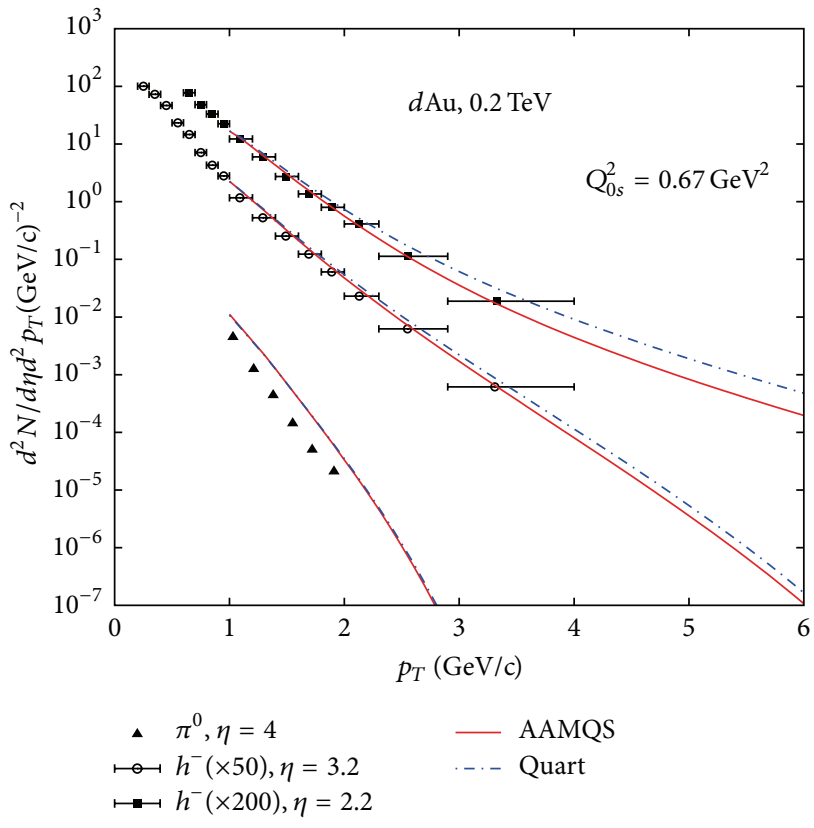

(b)

FIgURE 6: Same as Figure 3, but for minimum-bias $d+$ Au collisions with $Q_{0 s}^{2}=0.5 \mathrm{GeV}^{2}$ (a) and $0.67 \mathrm{GeV}^{2}$ (b) in the calculations.

represent the experimental data of the STAR [63] and BRAHMS collaborations [64] for different hadrons $\left(\pi^{0}[63]\right.$, $h^{-}$[64], and $\left.h^{-}[64]\right)$ at different pseudorapidities $(4.0,3.2$, and 2.2) with different magnifying multiples (1,50, and 200) in the distributions as shown in the figures. As we have already pointed out previously, the value of $Q_{0 s}$ at $x_{0}=$ 0.01 for proton target in the range $0.168 \div 0.336 \mathrm{GeV}^{2}$ gives a consistent description of the experimental data from the RHIC. We then choose $Q_{0 s}^{2}=0.168 \mathrm{GeV}^{2}$ (Figure 5(a)) and $Q_{0 s}^{2}=0.336 \mathrm{GeV}^{2}$ (Figure 5(b)) as input parameters for rcBK equation with the two different initial conditions. From Figure 5 we see that the results of the two initial conditions are similar to each other and approximately in agreement with the data.

In Figure 6, we compare our calculated results of $p_{T}$ distributions for $\pi^{0}$ and $h^{-}$produced in minimum-bias $d+\mathrm{Au}$ collisions at $\sqrt{s_{N N}}=0.2 \mathrm{TeV}$ with the experimental data of the STAR [63] and BRAHMS collaborations [64] at the RHIC. Except the interacting system and the values of $Q_{0 s}^{2}$, other illuminations are the same as those for Figure 5. According to $[27,57]$, the value of saturation scale for gold within $Q_{0 s}^{2}=$ $0.5 \div 0.67 \mathrm{GeV}^{2}$ is consistent with the RHIC data. It is difficult to pin down the exact value of $Q_{0 s}$ for nuclei since the DIS data for nuclear targets are limited and have large experimental uncertainties. In this case, we choose $Q_{0 s}^{2}=0.5 \mathrm{GeV}^{2}$ for Figure 6(a) and $Q_{0 s}^{2}=0.67 \mathrm{GeV}^{2}$ for Figure 6(b) as input parameters for rcBK equation with the two different initial conditions. We see that at $Q_{0 s}^{2}=0.5 \mathrm{GeV}^{2}$ the quartic action seems better and at $Q_{0 s}^{2}=0.67 \mathrm{GeV}^{2}$ the AAMQS seems better when we compare them with the experimental data of the STAR [63] and BRAHMS collaborations [64].
As shown in Figures 5 and 6, the single inclusive hadron production yields at different forward pseudorapidities in $p p$ and minimum-bias $d+A u$ collisions at the RHIC energy are approximately described by the $\mathrm{rcBK}$ dipole solution with the two different initial conditions. We notice that the two different initial conditions are matched together at very forward pseudorapidity $(\eta=4)$. However, the discrepancy goes large when the pseudorapidity goes small, which would be caused by the dynamical evolution with increasing the nuclear mass number.

We now present our predictions for single inclusive hadron production at the LHC in terms of the nuclear modification factor $R_{p A}$ and hope that some of the theoretical uncertainties, such as the sensitivity to $K$-factor, will be reduced. The nuclear modification factor $R_{p A}$ is defined as

$$
R_{p A}=\frac{1}{N_{\text {coll }}} \frac{d N^{p A \rightarrow h X} / d^{2} p_{T} d \eta}{d N^{p p \rightarrow h X} / d^{2} p_{T} d \eta},
$$

where $N_{\text {coll }}$ is the number of binary nucleon-nucleon collisions for a given centrality class in $p A$ collisions, and $d N^{p A \rightarrow h X} / d^{2} p_{T} d \eta$ and $d N^{p p \rightarrow h X} / d^{2} p_{T} d \eta$ denote the single inclusive hadron productions in $p A$ and $p p$ collisions, respectively. We take $N_{\text {coll }}=6.5$ and 7.4 for minimum-bias $p+\mathrm{Pb}$ collisions at $\sqrt{s_{N N}}=4.4$ and $8.8 \mathrm{TeV}$, respectively, according to a standard Monte Carlo Glauber model [65].

In Figure 7 we show the nuclear modification factor $R_{p A}^{c h}$ for inclusive charged hadrons $h^{+}+h^{-}$production in minimum-bias $p+\mathrm{Pb}(A=\mathrm{Pb}$ in $p A)$ collisions at $\sqrt{s_{N N}}=$ $4.4 \mathrm{TeV}$ (Figure 7(a) with $N_{\text {coll }}=6.5$ ) and $\sqrt{s_{N N}}=8.8 \mathrm{TeV}$ (Figure 7(b) with $N_{\text {coll }}=7.4$ ) with $\eta=4,5,6$, and 7 (from the upper curve to the lower one). All the results are obtained by 


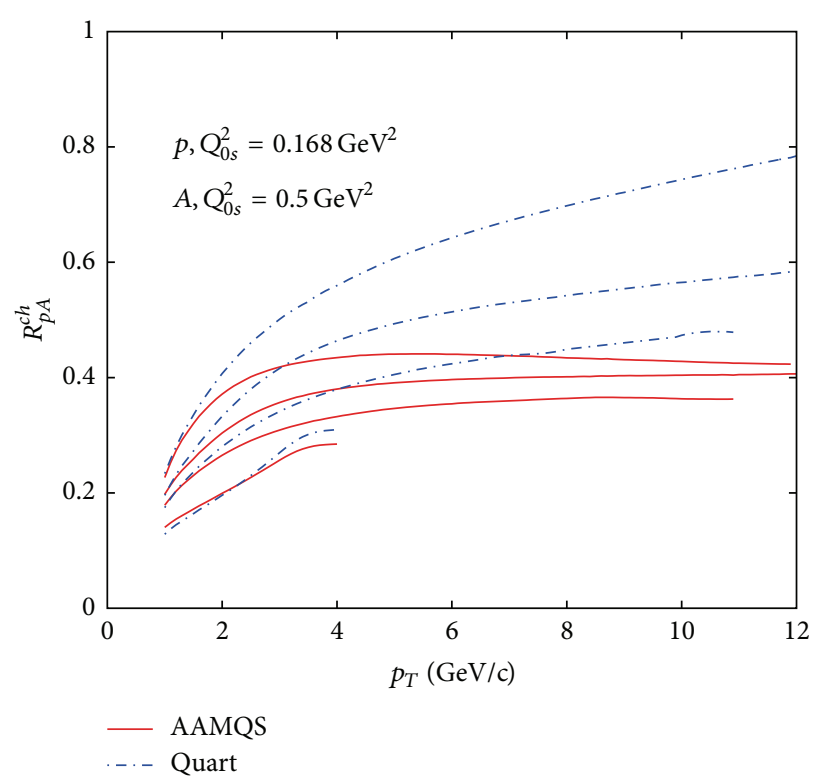

(a)

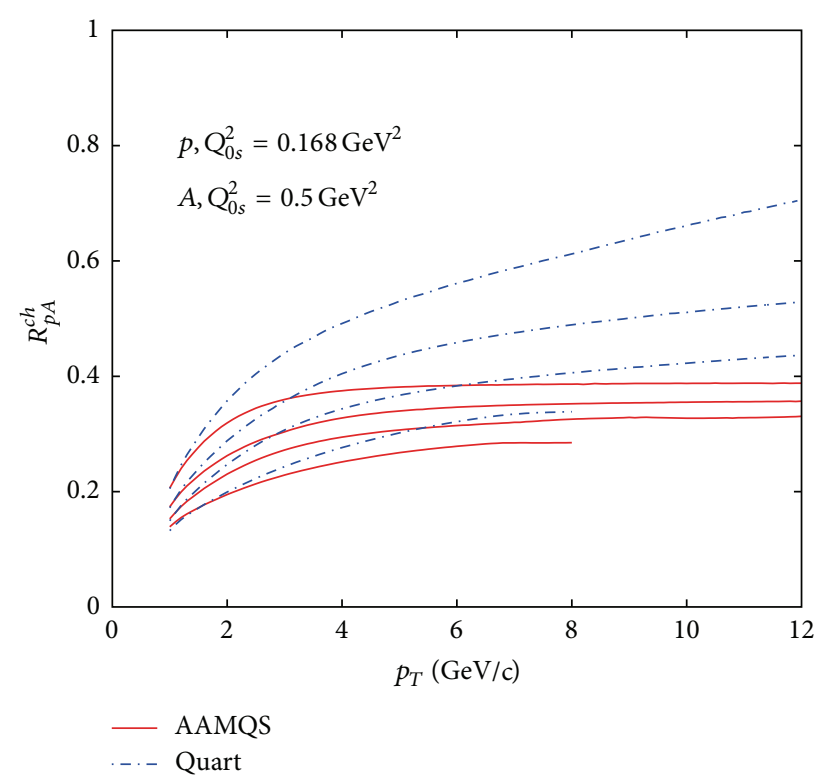

(b)

Figure 7: Nuclear modification factor $R_{p A}^{c h}$ versus $p_{T}$ for inclusive charged hadrons $h^{+}+h^{-}$production in minimum-bias $p+\mathrm{Pb}(A=\mathrm{Pb}$ in $p A)$ collisions at the LHC energies, with the pseudorapidity being 4, 5, 6, and 7 from the upper curve to lower one. The solid and dashed-dotted curves represent the results calculated by the AAMQS and quartic action, respectively, with $Q_{0 s}^{2}=0.168 \mathrm{GeV}^{2}$ for $p$ and $0.5 \mathrm{GeV}^{2}$ for $\mathrm{Pb}$ in the calculations. (a) corresponds to $\sqrt{s_{N N}}=4.4 \mathrm{TeV}$ with $N_{\text {coll }}=6.5$, and (b) corresponds to $\sqrt{s_{N N}}=8.8 \mathrm{TeV}$ with $N_{\text {coll }}=7.4$.

using the solution of the rcBK dipole evolution equation with two different initial conditions, AAMQS (the solid curves) and quartic action (the dashed-dotted curves). In both panels, we assume the initial saturation scales of proton and nucleus to be $Q_{0 s}^{2}=0.168 \mathrm{GeV}^{2}$ and $Q_{0 s}^{2}=0.5 \mathrm{GeV}^{2}$, respectively. From Figure 7 one can see that $R_{p A}^{c h}$ decreases with increasing the pseudorapidity. This is explained from nonlinear evolution which suppresses additional gluon emissions in dense wave functions as compared to the dilute limit. The relationship between $R_{p A}^{c h}$ and $\sqrt{s_{N N}}$ is weak in the LHC energy region. We note also from Figure 7 that the quartic action as the solution of $\mathrm{rCBK}$ equation leads to a faster rise of $R_{p A}^{c h}$ with transverse momentum, and the corresponding behavior is obviously different from AAMQS. This is because the particle production at high $p_{T}$ in $p A$ collisions is mainly dominated by a high valence color charge density in the dense target.

Because the DIS data for nuclear targets are limited and have large experimental uncertainties, we calculate the possible ranges of $R_{p A}^{c h}$ for forward rapidities in minimum-bias $p+\mathrm{Pb}$ collisions at two energies using two initial saturation momentum scales with two initial conditions, AAMQS (the double solid curves) and quartic action (the double dasheddotted curves), in Figure $8\left(\sqrt{s_{N N}}=4.4 \mathrm{TeV}\right)$ and Figure 9 $\left(\sqrt{s_{N N}}=8.8 \mathrm{TeV}\right)$, respectively. The lower curves correspond to $Q_{0 s}^{2}=0.5 \mathrm{GeV}^{2}$ and the upper curves correspond to $Q_{0 s}^{2}=0.67 \mathrm{GeV}^{2}$ for nuclear target. We note that there are large uncertainties in $R_{p A}^{c h}$ at small pseudorapidity at the LHC due to the choice of the initial saturation scale for the rcBK evolution equation. In particular, if we choose the special non-Gaussian action (quartic action) which involves the subleading correction of the valence color charges, the discrepancy will be clearly larger compared to the other one (AAMQS).

It should be noted that the particle production crosssection given by (13) is right at forward rapidity. If we calculate the particle production at central rapidity, we need another formula, the $k_{t}$-factorization formalism [66-68]. This will dilute our comparison on the two initial conditions. To give prominence to the comparison, we have used only the hybrid formalism (13) at forward rapidity in this paper.

\section{Conclusions}

In this work we have used quantitatively a new initial condition (the quartic action initial condition) as input for the $\mathrm{rcBK}$ equation $[18,19]$ and presented a description of the hadron yields measured at three different pseudorapidities in $p p$ and $d+A u$ collisions at the RHIC by using the hybrid formalism which is proposed in [58]. The results calculated by the quartic action and AAMQS models [36-38] have been compared to each other and some interesting results have been obtained.

At medium and large $r Q_{s}\left(x_{0}\right)$, the quartic action gives a comparable result of the scattering amplitude for an adjoint dipole with the MV $[41,42]$ and AAMQS models. At small $r Q_{s}\left(x_{0}\right)$, the quartic action gives a much lower scattering amplitude which is beyond the MV and AAMQS models. The results on the proton structure function $F_{2}\left(x, Q^{2}\right)$ calculated 


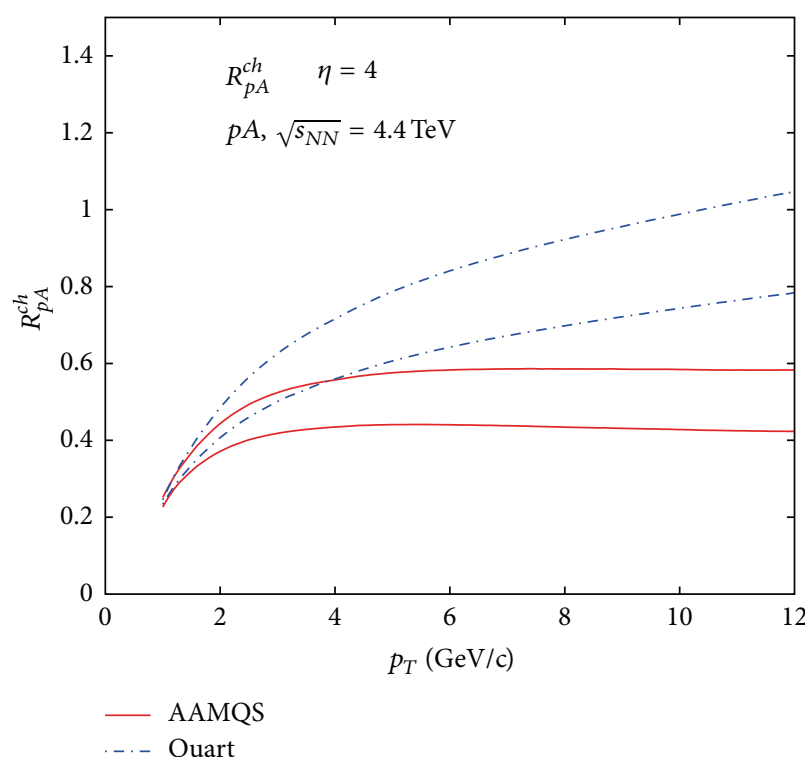

(a)

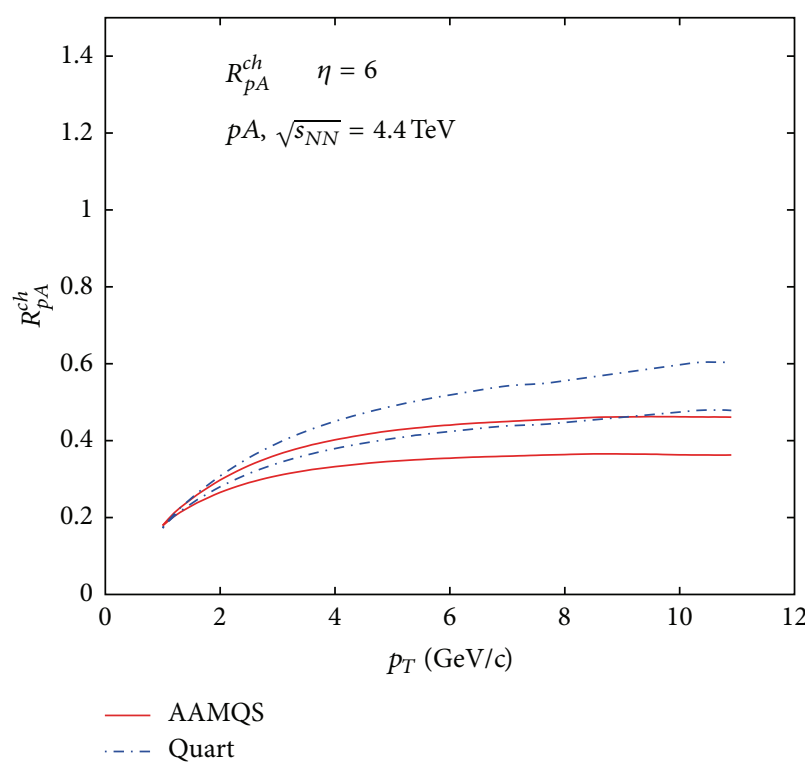

(c)

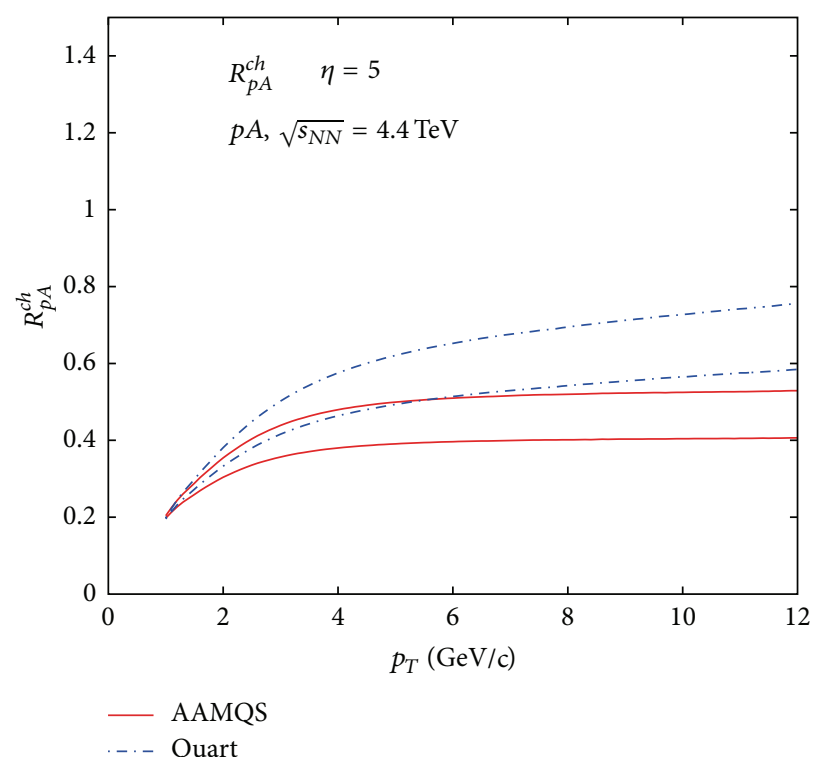

(b)

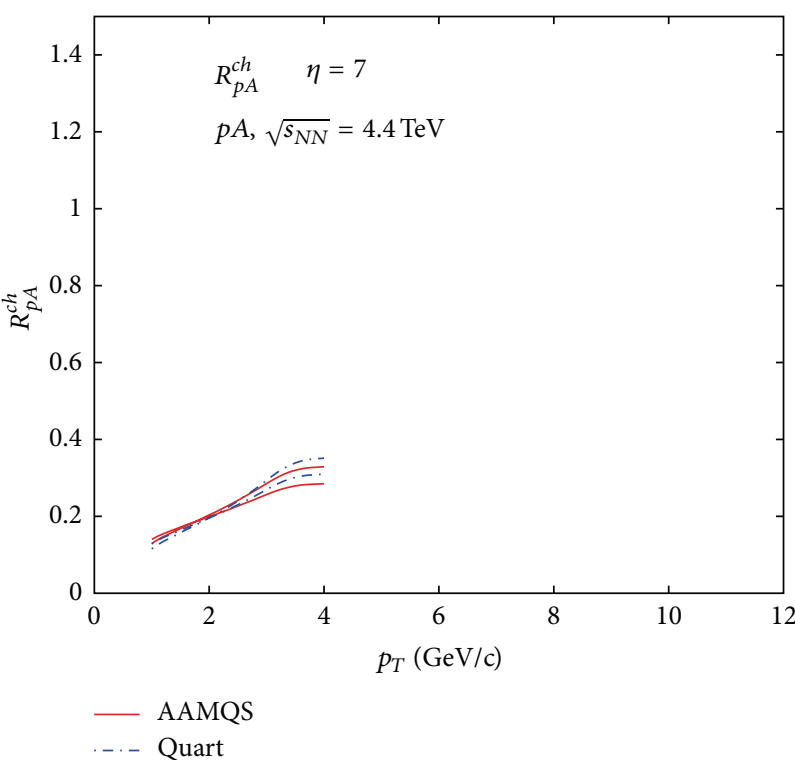

(d)

FIGURE 8: Nuclear modification factor $R_{p A}^{c h}$ versus $p_{T}$ for inclusive charged hadrons $h^{+}+h^{-}$production in minimum-bias $p+\mathrm{Pb}$ collisions at $\sqrt{s_{N N}}=4.4 \mathrm{TeV}$ with different $\eta$. The double solid curves and the double dashed-dotted curves are calculated, respectively, by the AAMQS and quartic action, which represents the uncertainty ranges in $R_{p A}^{c h}$ when $Q_{0 s}^{2}=0.5$ to $0.67 \mathrm{GeV}^{2}$.

by the quartic action and AAMQS models are very similar and describe approximately the HERA data [53-55]. In a few cases, for example, $Q^{2}=8.5 \mathrm{GeV}^{2}$, the two models present almost the same results on $F_{2}\left(x, Q^{2}\right)$.

In the description of hadron transverse momentum distributions in $p p$ collisions at the RHIC energy, both quartic action and AAMQS models present almost the same results at forward pseudorapidity. At the closely mid-pseudorapidity, the results calculated by the two models are similar. In $d+\mathrm{Au}$ collisions at the RHIC energy, both of the two models present almost the same results at forward pseudorapidity on the
$p_{T}$ distribution. At the closely mid-pseudorapidity, the two modeling results have an obvious difference in the high $p_{T}$ region, which is caused by the dynamical evolution with increasing the nuclear mass number.

Based on the comparisons with the experimental data at the RHIC energy, the nuclear modification factor $R_{p A}^{c h}$ for charged hadrons produced in $p A$ collisions at the LHC energies is predicted by using the previously mentioned two models. The calculated results show that $R_{p A}^{c h}$ decreases with increasing the pseudorapidity, and a large uncertainty in $R_{p A}^{c h}$ 


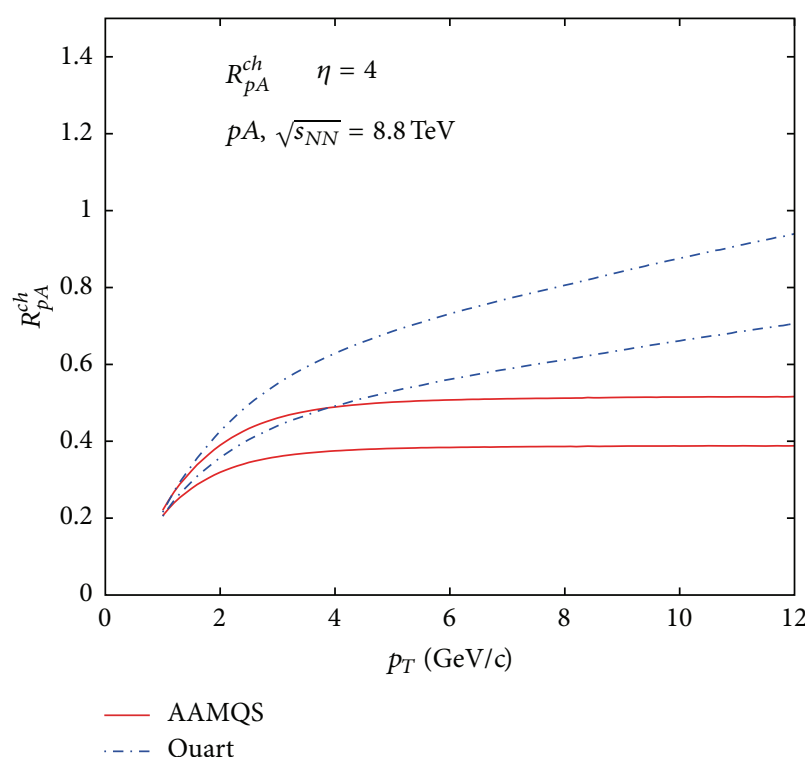

(a)

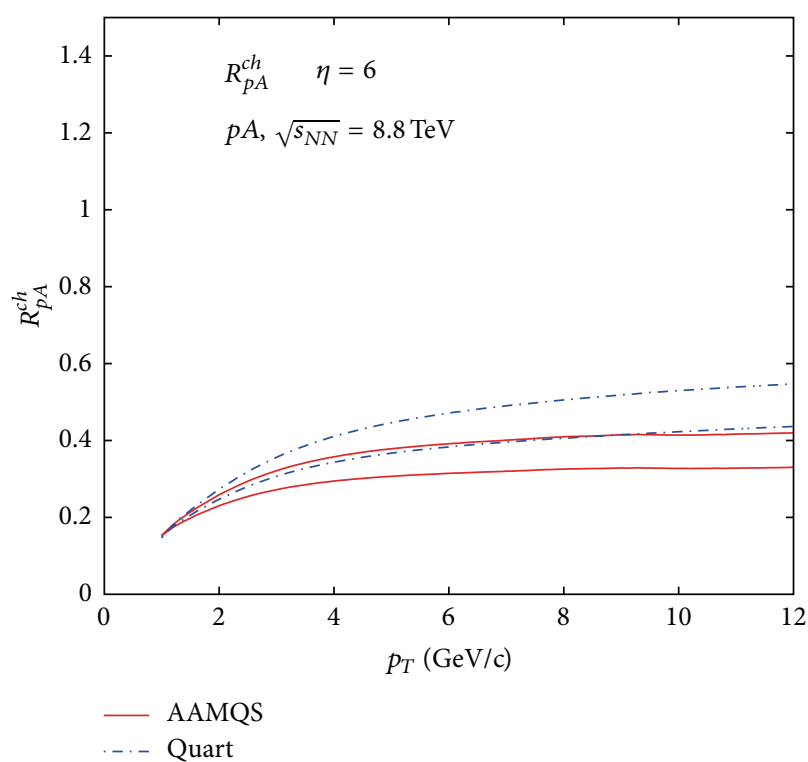

(c)

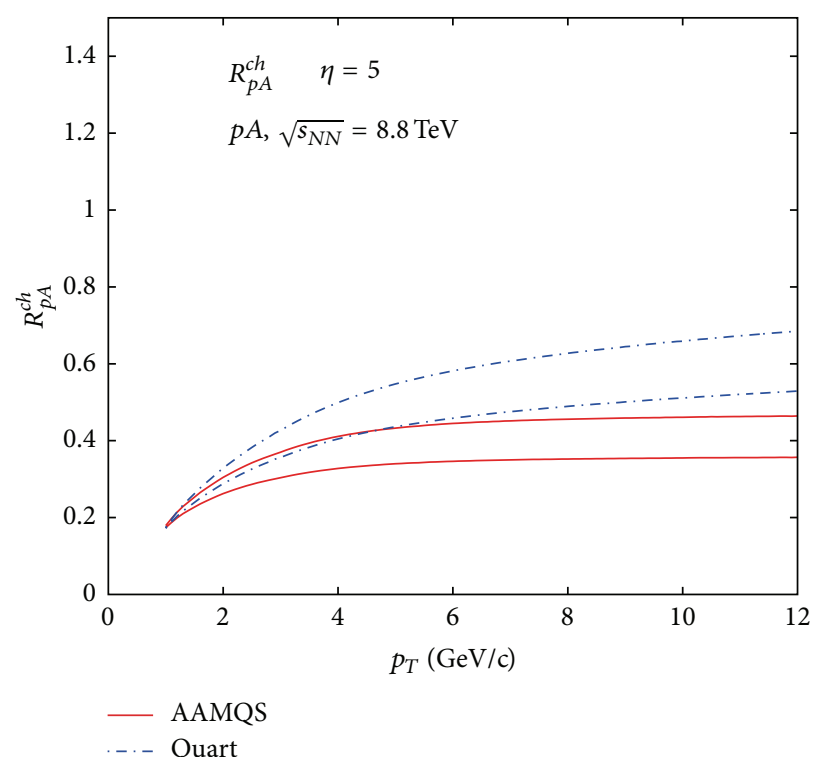

(b)

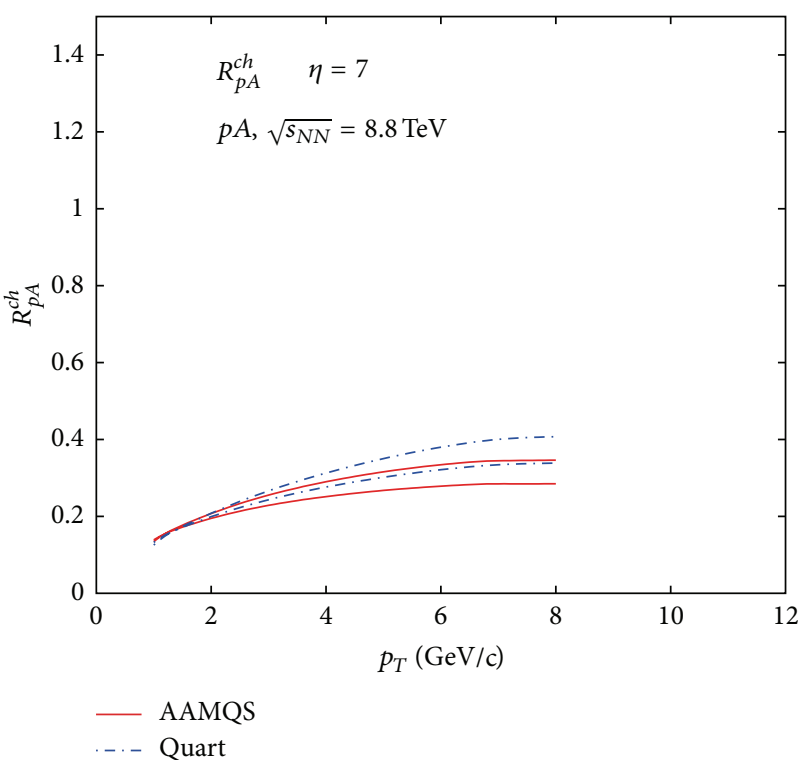

(d)

Figure 9: Same as Figure 8, but for the results at $\sqrt{s_{N N}}=8.8 \mathrm{TeV}$.

at small pseudorapidity is obtained. The quartic action as the solution of rcBK equation leads to a faster rise of $R_{p A}^{c h}$ with transverse momentum, and the quartic action shows a larger uncertainty than the AAMQS. These behaviors are related to the correlation and density fluctuation of the valence color charges. Our calculated results in the present work are consistent with the results given in recent works $[25,69]$.

\section{Acknowledgments}

This work was supported by the National Natural Science Foundation of China (10975095), the China National Fundamental Fund of Personnel Training (J1103210), the Open
Research Subject of the Chinese Academy of Sciences LargeScale Scientific Facility (2060205), and the Shanxi Scholarship Council of China.

\section{References}

[1] F. Gelis, E. Iancu, J. Jililian-Marian, and R. Venugopalan, "The color glass condensate," Annual Review of Nuclear and Particle Science, vol. 60, pp. 463-489, 2010.

[2] J. Jalilian-Marian and Y. V. Kovchegov, "Saturation physics and deuteron-gold collisions at RHIC," Progress in Particle and Nuclear Physics, vol. 56, no. 1, pp. 104-231, 2006.

[3] J. Jalilian-Marian, A. Kovner, A. Leonidov, and H. Weigert, "Wilson renormalization group for low x physics: towards the 
high density regime," Physical Review D, vol. 59, no. 1, Article ID 014014, 6 pages, 1999.

[4] J. Jalilian-Marian, A. Kovner, and H. Weigert, "Wilson renormalization group for low x physics: gluon evolution at finite parton density," Physical Review D, vol. 59, no. 1, Article ID 014015, 24 pages, 1999.

[5] A. Kovner, J. G. Milhano, and H. Weigert, "Relating different approaches to nonlinear QCD evolution at finite gluon density," Physical Review D, vol. 62, no. 11, Article ID 114005, 24 pages, 2000.

[6] H. Weigert, "Unitarity at small Bjorken $x$," Nuclear Physics A, vol. 703, no. 3-4, pp. 823-860, 2002.

[7] E. Iancu, A. Leonidov, and L. McLerran, "Nonlinear gluon evolution in the color glass condensate: I," Nuclear Physics A, vol. 692, no. 3-4, pp. 583-645, 2001.

[8] E. Ferreiro, E. Iancu, A. Leonidov, and L. McLerran, "Nonlinear gluon evolution in the color glass condensate: II," Nuclear Physics A, vol. 703, no. 1-2, pp. 489-538, 2002.

[9] N. N. Nikolaev and B. G. Zakharov, "Colour transparency and scaling properties of nuclear shadowing in deep inelastic scattering," Zeitschrift für Physik C, vol. 49, no. 4, pp. 607-618, 1991.

[10] A. H. Mueller, "Small- $x$ behavior and parton saturation: a QCD model," Nuclear Physics B, vol. 335, no. 1, pp. 115-137, 1990.

[11] I. Balitsky, "Operator expansion for high-energy scattering," Nuclear Physics B, vol. 463, no. 1, pp. 99-157, 1996.

[12] Y. V. Kovchegov, "Small- $x F_{2}$ structure function of a nucleus including multiple Pomeron exchanges," Physical Review D, vol. 60, no. 3, Article ID 034008, 8 pages, 1999.

[13] Y. V. Kovchegov, "Unitarization of the BFKL Pomeron on a nucleus," Physical Review D, vol. 61, no. 7, Article ID 074018, 15 pages, 2000.

[14] I. Balitsky, "Quark contribution to the small- $x$ evolution of color dipole," Physical Review D, vol. 75, no. 1, Article ID 014001, 21 pages, 2007.

[15] Y. V. Kovchegov and H. Weigert, "Triumvirate of running couplings in small- $x$ evolution," Nuclear Physics A, vol. 784, no. 1-4, pp. 188-226, 2007.

[16] E. Gardi, J. Kuokkanen, K. Rummukainen, and H. Weigert, "Running coupling and power corrections in non-linear evolution at the high-energy limit," Nuclear Physics A, vol. 784, no. 1-4, pp. 282-340, 2007.

[17] I. Balitsky and G. A. Chirilli, "Next-to-leading order evolution of color dipoles," Physical Review D, vol. 77, no. 1, Article ID 014019, 43 pages, 2008.

[18] J. L. Albacete and Y. V. Kovchegov, "Solving the high energy evolution equation including running coupling corrections," Physical Review D, vol. 75, no. 12, Article ID 125021, 17 pages, 2007.

[19] J. L. Albacete, "Particle multiplicities in lead-lead collisions at the CERN large hadron collider from nonlinear evolution with running coupling corrections," Physical Review Letters, vol. 99, no. 26, Article ID 262301, 4 pages, 2007.

[20] D. Kharzeev, Y. V. Kovchegov, and K. Tuchin, "Nuclear modification factor in $d+$ Au collisions: onset of suppression in the color glass condensate," Physics Letters B, vol. 599, no. 1-2, pp. 23-31, 2004.

[21] K. Tuchin, "Forward hadron production in high energy $p A$ collisions: from RHIC to LHC," Nuclear Physics A, vol. 798, no. 1-2, pp. 61-73, 2008.
[22] J. Jalilian-Marian, "Forward rapidity hadron production in deuteron-gold collisions from valence quarks," Nuclear Physics A, vol. 748, no. 3-4, pp. 664-671, 2005.

[23] A. Dumitru, A. Hayashigaki, and J. Jalilian-Marian, "The color glass condensate and hadron production in the forward region," Nuclear Physics A, vol. 765, no. 3-4, pp. 464-482, 2006.

[24] A. Dumitru, A. Hayashigaki, and J. Jalilian-Marian, "Geometric scaling violations in the central rapidity region of $d+\mathrm{Au}$ collisions at RHIC," Nuclear Physics A, vol. 770, no. 1-2, pp. 5770, 2006.

[25] J. L. Albacete, A. Dumitru, H. Fujii, and Y. Nara, "CGC predictions for $p+\mathrm{Pb}$ collisions at the LHC," Nuclear Physics A, vol. 897, pp. 1-27, 2013.

[26] V. P. Gonçalves, M. S. Kugeratski, M. V. T. Machado, and F. S. Navarra, "Saturation physics at HERA and RHIC: an unified description," Physics Letters B, vol. 643, no. 5, pp. 273-278, 2006.

[27] J. L. Albacete and C. Marquet, "Single inclusive hadron production at RHIC and the LHC from the color glass condensate," Physics Letters B, vol. 687, no. 2-3, pp. 174-179, 2010.

[28] A. H. Rezaeian and A. Schäfer, "Hadrons and direct photon production in $p p$ and $p A$ collisions at the LHC and saturation effects," Physical Review D, vol. 81, no. 11, Article ID 114032, 22 pages, 2010.

[29] H. Fujii, K. Itakura, Y. Kitadono, and Y. Nara, "Forward particle productions at the RHIC and the LHC from CGC within local rcBK evolution," Journal of Physics G, vol. 38, no. 12, Article ID 124125, 5 pages, 2011.

[30] R. B. Neufeld, I. Vitev, and B. W. Zhang, "A possible determination of the quark radiation length in cold nuclear matter," Physics Letters B, vol. 704, no. 5, pp. 590-595, 2011.

[31] N. Armesto, N. Borghini, S. Jeon et al., "Heavy-ion collisions at the LHC-last call for predictions," Journal of Physics G, vol. 35, no. 5, Article ID 054001, 2008.

[32] C. Marquet, "Forward inclusive dijet production and azimuthal correlations in $p A$ collisions," Nuclear Physics A, vol. 796, no. 14, pp. 41-60, 2007.

[33] J. L. Albacete and C. Marquet, "Azimuthal correlations of forward dihadrons in $d+$ Au collisions at RHIC in the color glass condensate," Physical Review Letters, vol. 105, no. 16, Article ID 162301, 4 pages, 2010.

[34] A. Stasto, B. W. Xiao, and F. Yuan, "Back-to-back correlations of di-hadrons in dAu collisions at RHIC," http://arxiv .org/abs/1109.1817.

[35] E. Avsar, A. M. Stasto, D. N. Triantafyllopoulos, and D. Zaslavsky, "Next-to-leading and resummed BFKL evolution with saturation boundary," http://arxiv.org/abs/1107.1252 .

[36] J. L. Albacete, N. Armesto, J. G. Milhano, and C. A. Salgado, "Nonlinear QCD meets data: a global analysis of leptonproton scattering with running coupling balitsky-Kovchegov evolution," Physical Review D, vol. 80, no. 3, Article ID 034031, 11 pages, 2009.

[37] J. L. Albacete, N. Armesto, J. G. Milhano, P. Quiroga Arias, and C. A. Salgado, "AAMQS: a non-linear QCD analysis of new HERA data at small- $x$ including heavy quarks," European Physical Journal C, vol. 71, no. 7, Article ID 1705, 2011.

[38] J. Albacete, J. Milhano, P. Quiroga-Arias, and J. Rojo, "Linear vs non-linear QCD evolution: from HERA data to LHC phenomenology," http://arxiv.org/abs/1203.1043 .

[39] J. Kuokkanen, K. Rummukainen, and H. Weigert, "HERA-data in the light of small $x$ evolution with state of the art NLO input," Nuclear Physics A, vol. 875, pp. 29-93, 2012. 
[40] A. Dumitru and E. Petreska, "Initial conditions for dipole evolution beyond the McLerran-Venugopalan model," Nuclear Physics A, vol. 879, pp. 59-76, 2012.

[41] L. McLerran and R. Venugopalan, "Computing quark and gluon distribution functions for very large nuclei," Physical Review D, vol. 49, no. 5, pp. 2233-2241, 1994.

[42] L. McLerran and R. Venugopalan, "Gluon distribution functions for very large nuclei at small transverse momentum," Physical Review D, vol. 49, no. 7, pp. 3352-3355, 1994.

[43] P. Tribedy and R. Venugopalan, "Saturation models of HERA DIS data and inclusive hadron distributions in $p+p$ collisions at the LHC," Nuclear Physics A, vol. 850, no. 1, pp. 136-156, 2011.

[44] S. Jeon and R. Venugopalan, "Random walks of partons in $\mathrm{SU}\left(N_{c}\right)$ and classical representations of color charges in QCD at small x," Physical Review D, vol. 70, no. 10, Article ID 105012, 17 pages, 2004.

[45] S. Jeon and R. Venugopalan, "Classical odderon in QCD at high energies," Physical Review D, vol. 71, no. 12, Article ID 125003, 7 pages, 2005.

[46] A. Dumitru, J. Jalilian-Marian, and E. Petreska, "Two-gluon correlations and initial conditions for small $x$ evolution," Physical Review D, vol. 84, no. 1, Article ID 014018, 9 pages, 2011.

[47] J. L. Albacete and A. Dumitru, "A model for gluon production in heavy-ion collisions at the LHC with rcBK unintegrated gluon densities," http://arxiv.org/abs/1011.5161 .

[48] A. Dumitru, "Phenomenology of high gluon density QCD and heavy-ion physics at ISMD 2011: $x$ smaller than ever !" Progress of Theoretical Physics Supplement. In press, http://arxiv.org/ abs/1112.3081.

[49] K. Dusling, F. Gelis, T. Lappi, and R. Venugopalan, "Long range two-particle rapidity correlations in $A+A$ collisions from high energy QCD evolution," Nuclear Physics A, vol. 836, no. 1-2, pp. 159-182, 2010.

[50] V. Barone and E. Predazzi, High-Energy Particle Diffraction, Springer, New York, NY, USA, 1st edition, 2002.

[51] H. G. Dosch, T. Gousset, G. Kulzinger, and H. J. Pirner, "Vector meson leptoproduction and nonperturbative gluon fluctuations in QCD," Physical Review D, vol. 55, no. 5, pp. 2602-2615, 1997.

[52] K. Golec-Biernat and M. Wüsthoff, "Saturation effects in deep inelastic scattering at low $Q^{2}$ and its implications on diffraction," Physical Review D, vol. 59, no. 1, Article ID 014017, 10 pages, 1998.

[53] S. Aid, V. Andreev, B. Andrieu et al., "A measurement and QCD analysis of the proton structure function $F_{2}\left(x, Q^{2}\right)$ at HERA," Nuclear Physics B, vol. 470, no. 1-2, pp. 3-38, 1996.

[54] T. Ahmed, S. Aid, A. Akhundov et al., "A measurement of the proton structure function $F_{2}\left(x, Q^{2}\right)$," Nuclear Physics B, vol. 439, no. 3, pp. 471-502, 1995.

[55] M. Derrick, D. Krakauer, S. Magill et al., "Measurement of the proton structure function $F_{2}$ at low $x$ and low $Q^{2}$ at HERA," Zeitschrift für Physik C, vol. 69, no. 1, pp. 607-620, 1996.

[56] D. de Florian, R. Sassot, and M. Stratmann, "Global analysis of fragmentation functions for protons and charged hadrons," Physical Review D, vol. 76, no. 7, Article ID 074033, 16 pages, 2007.

[57] J. Jalilian-Marian and A. H. Rezaeian, "Hadron production in $p A$ collisions at the LHC from the color glass condensate," Physical Review D, vol. 85, no. 1, Article ID 014017, 10 pages, 2012.

[58] T. Altinoluk and A. Kovner, "Particle production at high energy and large transverse momentum: "The hybrid formalism" revisited," Physical Review D, vol. 83, no. 10, Article ID 105004, 13 pages, 2011.
[59] G. Altarelli and G. Parisi, "Asymptotic freedom in parton language," Nuclear Physics B, vol. 126, no. 2, pp. 298-318, 1977.

[60] K. Nakamura, K. Hagiwara, K. Hikasa et al., "Review of particle physics," Journal of Physics G, vol. 37, no. 7, Article ID 075021, 2010.

[61] H.-L. Lai, M. Guzzi, J. Huston et al., "New parton distributions for collider physics," Physical Review D, vol. 82, no. 7, Article ID 074024, 24 pages, 2010.

[62] D. de Florian, R. Sassot, and M. Stratmann, "Global analysis of fragmentation functions for pions and kaons and their uncertainties," Physical Review D, vol. 75, no. 11, Article ID 114010, 26 pages, 2007.

[63] J. Adams, M. M. Aggarwal, Z. Ahammed et al., “ Forward neutral pion production in $p+p$ and $d+$ Au collisions at $\sqrt{s_{N N}}=200 \mathrm{GeV}$," Physical Review Letters, vol. 97, no. 15, Article ID 152302, 6 pages, 2006.

[64] I. Arsene, I. G. Bearden, D. Beavis et al., "Evolution of the nuclear modification factors with rapidity and centrality in $d+$ Au collisions at $\sqrt{s_{N N}}=200 \mathrm{GeV}$," Physical Review Letters, vol. 93, no. 24, Article ID 242303, 4 pages, 2004.

[65] D. d'Enterria, "Hard scattering cross sections at LHC in the Glauber approach: from $p p$ to $p A$ and $A A$ collisions," http://arxiv.org/abs/nucl-ex/0302016 .

[66] Y. V. Kovchegov and K. Tuchin, "Inclusive gluon production in deep inelastic scattering at high parton density," Physical Review D, vol. 65, no. 7, Article ID 074026, 16 pages, 2002.

[67] W. A. Horowitz and Y. V. Kovchegov, "Running coupling corrections to high energy inclusive gluon production," Nuclear Physics A, vol. 849, no. 1, pp. 72-97, 2011.

[68] F. Gelis and R. Venugopalan, "Large mass $q \bar{q}$ production from the color glass condensate," Physical Review D, vol. 69, no. 1, Article ID 014019, 13 pages, 2004.

[69] A. H. Rezaeian, "CGC predictions for $p+A$ collisions at the LHC and signature of QCD saturation," Physics Letters B, vol. 718, no. 3, pp. 1058-1069, 2013. 

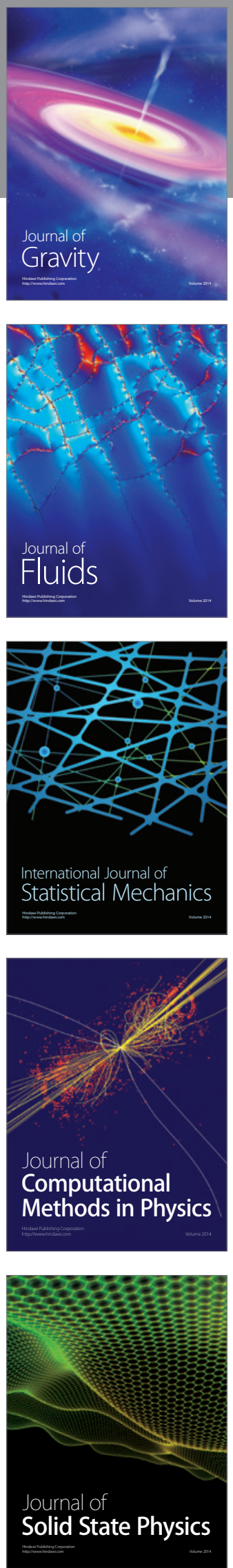

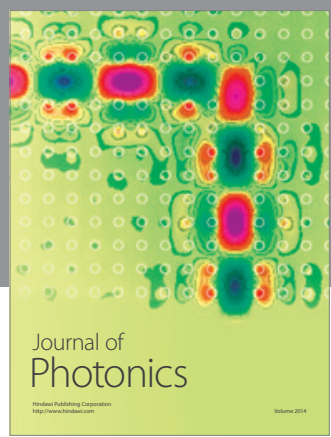

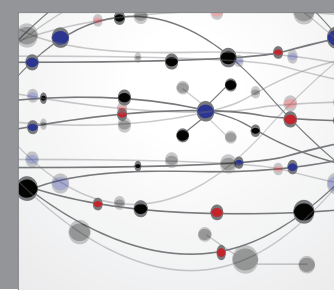

The Scientific World Journal

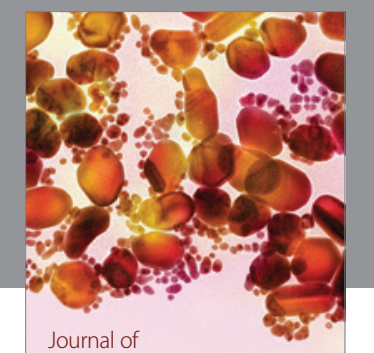

Soft Matter
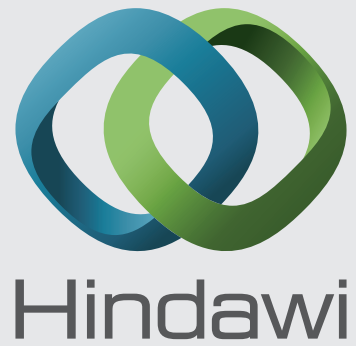

Submit your manuscripts at

http://www.hindawi.com
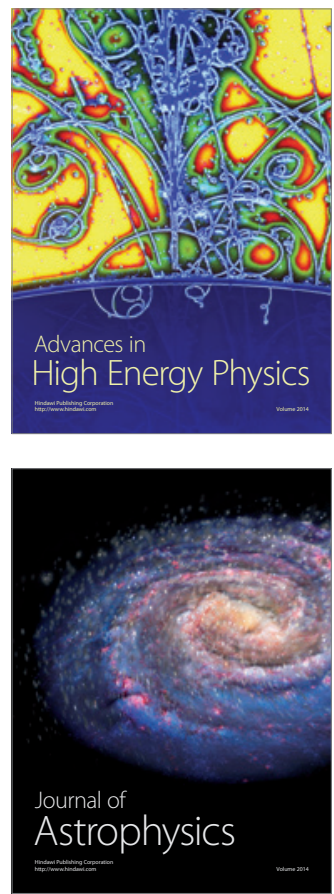
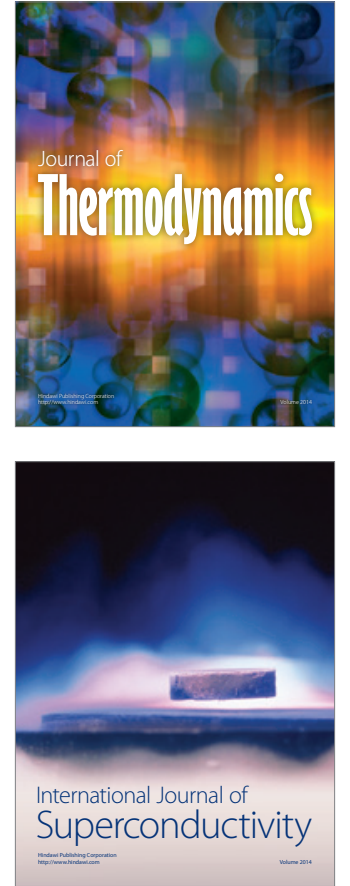
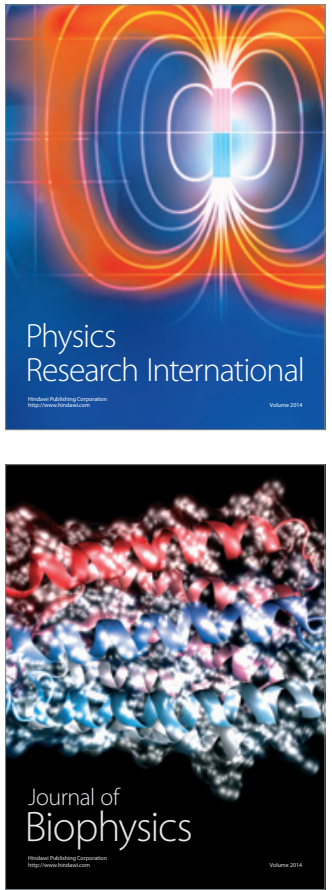
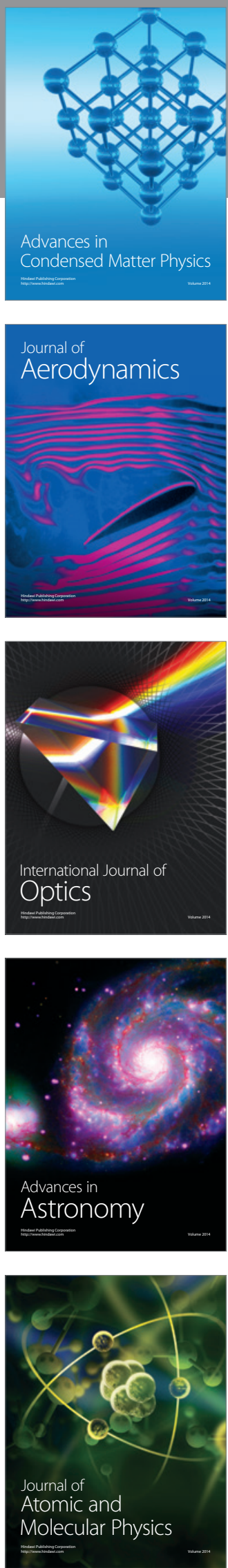